\title{
Measurement and implications of Saturn's gravity field and ring mass
}

Authors: L. Iess ${ }^{1 *}$, B. Militzer ${ }^{2}$, Y. Kaspi ${ }^{3}$, P. Nicholson ${ }^{4}$, D. Durante ${ }^{1}$, P. Racioppa ${ }^{1}$, A. Anabtawi $^{5}$, E. Galanti ${ }^{3}$, W. Hubbard ${ }^{6}$, M. J. Mariani ${ }^{1}$, P. Tortora ${ }^{7}$, S. Wahl ${ }^{2}$, M. Zannoni ${ }^{7}$

\author{
Affiliations: \\ ${ }^{1}$ Sapienza Università di Roma, Rome 00184, Italy \\ ${ }^{2}$ University of California, Berkeley, CA 94720, USA \\ ${ }^{3}$ Weizmann Institute of Science, Rehovot 76100, Israel \\ ${ }^{4}$ Cornell University, Ithaca, NY 14853, United States \\ ${ }^{5}$ Jet Propulsion Laboratory/Caltech, Pasadena, CA 91109, USA \\ ${ }^{6}$ Lunar and Planetary Laboratory, University of Arizona, Tucson, AZ 85721, USA \\ ${ }^{7}$ Università di Bologna, Forlì 47100, Italy \\ *Correspondence to: luciano.iess@uniroma1.it
}

\begin{abstract}
:
The interior structure of Saturn, the depth of its winds and the mass and age of its rings constrain its formation and evolution. In the final phase of the Cassini mission, the spacecraft dived between the planet and the innermost ring, at altitudes $2600-3900 \mathrm{~km}$ above the cloud tops. During six of these crossings, a radio link with Earth was monitored to determine the gravitational field of the planet and the mass of its rings. We find that Saturn's gravity deviates from theoretical expectations and requires differential rotation of the atmosphere extending to a depth of at least $9000 \mathrm{~km}$. The total mass of the rings is $0.41 \pm 0.13$ of the Saturnian moon Mimas, indicating that they may have formed only $10^{7}-10^{8}$ years ago.
\end{abstract}

\section{One Sentence Summary:}

Measurements of the radial velocity of the Cassini spacecraft indicate a strong differential rotation inside the planet, a substantial core and a low mass - and thus a young age - for its rings.

\section{Main text:}

The mass distribution inside a fluid and rapidly rotating planet, such as Saturn, is largely driven by the ratio between centrifugal and gravity forces. In the absence of internal dynamics, axial and hemispherical symmetry is expected, implying that in the decomposition of the gravitational potential into spherical harmonics (an orthonormal basis for functions defined over the sphere) only even zonal harmonics appear (zonal harmonics are longitude-independent). Assuming hydrostatic equilibrium, interior models of gas giant planets indicate that the zonal coefficients $J_{2 k}$ can be approximated by $J_{2 k}=a_{k} q^{k}$, where $q$ is the ratio of the centrifugal and gravity 
acceleration at the equator (about 0.16 for Saturn), $k$ is an integer positive number, and the coefficients $a_{k}$ depend on the density profile inside the planet (1).

Optical tracking of clouds indicates that dynamical phenomena operate on Saturn and Jupiter. The measured zonal (west-east) wind velocity field suggests a state of differential rotation, whereby the angular velocity at any location depends on its distance from the axis of rotation and the depth along this axis $(2,3)$. If the velocity field seen at the cloud top level (conventionally defined as the 1 bar level) continues into the interior, then internal dynamics are expected to affect the gravitational field in two ways. Firstly, the equipotential surfaces are perturbed symmetrically, redistributing mass in such a way that the even zonal coefficients deviate from the relation $J_{2 k} \sim q^{k}(2)$. Secondly, any north-south asymmetry in the velocity field leads to nonzero values of the odd zonal harmonics (4). These theoretical expectations have been confirmed by the Juno mission at Jupiter (5-7), where gravity measurements showed that zonal winds are 2000-3000 km deep and suggest that the heavy element core is diffuse (8).

Gravity measurements at Saturn can be used to determine the mass of the rings, which dynamical and compositional dating methods show is related to the rings' age (9-11). Prior to the Grand Finale phase of the mission, the pericenter of Cassini's orbit was always outside Saturn's A ring, so that the gravitational effects of the rings could not be separated from those of the oblateness of the planet. During the Grand Finale, Cassini flew between the planet and the rings. This geometry effectively breaks the degeneracy between the even zonal field and the mass of the rings, providing a direct, dynamical estimate of the ring mass.

\section{Cassini gravity measurements}

We determined Saturn's gravitational field by reconstruction of Cassini's trajectory during the Grand Finale, using a coherent microwave link between Earth tracking stations and the spacecraft. Range-rate measurements were obtained from the Doppler shift of a carrier signal sent from the ground at 7.2 GHz (X-band) and retransmitted back to Earth by Cassini's onboard transponder at $8.4 \mathrm{GHz}$. An auxiliary downlink at Ka-band $(32.5 \mathrm{GHz})$ was also recorded.

In April 2017, Cassini was inserted into a series of inclined, highly eccentric orbits, grazing Saturn's cloud tops at each pericenter (Table S1). The orbit nodes were chosen such that the angle between the orbit normal and the direction to Earth is close to $90^{\circ}$. This edge-on condition provides the maximum projection of the spacecraft velocity along the line of sight, so is optimal for range-rate measurements and gravity estimations.

Of the 22 Grand Finale orbits (labelled Rev 271 through Rev 293), six were selected for gravity measurements. Five orbits (Revs 273, 274, 278, 280 and 284) provided useful data (data from Rev 275 were lost due to a station configuration error). These orbits were selected to minimize neutral particle drag and maximize spacecraft view period around closest approach (C/A).

We produced an orbital solution based on 5 data arcs, using Doppler observations with count times of $30 \mathrm{~s}$, spanning a period of 24-36 hours about each closest approach. Tracking data were acquired by the antennas of the three complexes (Goldstone, USA; Madrid, Spain; and Canberra, Australia) of NASA's Deep Space Network (DSN), and two deep space antennas from ESA's ESTRACK network, located in the southern hemisphere (Malargüe, Argentina, and New Norcia, Australia). 
Two-way Doppler measurements at X-band make up 93\% of the data set (12), with the addition of a few three-way passes to fill gaps during ground station handovers. Available X-band range observables are also included. All data with either uplink or downlink elevation below $15^{\circ}$ were discarded to avoid systematic measurement errors due to imperfect calibration of tropospheric path delays. Calibrations of Earth's tropospheric and ionospheric path delays were provided by the DSN based on pressure, humidity and Global Positioning System data. Noise from solar plasma, which can strongly affect X-band radio links, was low due to the large solar elongation angle ( $>142^{\circ}$ on all 6 gravity orbits) (13). The data quality was statistically equivalent in X-and

Ka-band data, with a root-mean-square (RMS) Doppler noise between 0.020 and $0.088 \mathrm{~mm} \cdot \mathrm{s}^{-1}$ at $30 \mathrm{~s}$ integration time (Fig. S1 and Table S1). For comparison, the Doppler signals due to the weakest measurable harmonics $\left(J_{3}, J_{10}\right)$ and Saturn's ring are 40-200 times larger than the average Doppler noise (Fig. S2).

The X-band Doppler data were favoured in our analysis because of the higher signal-to-noise ratio during the unavoidable ring occultation periods. The radio link was maintained during the occultations, except for a blockage of about 10 minutes on each orbit when the signal crossed the opaque core of the optically thick B ring (region B3). A second, longer blockage period due to ring occultations occurred on the outbound leg of the gravity orbits. Diffraction and near-forward scattering of the radio signal by ring particles caused an increase in the Doppler noise at X-band by a factor of 2-3 during the occultation periods. Ka-band is more sensitive to this effect and suffers repeated signal losses.

\section{Dynamical model}

Our orbital fitting is based on the dynamical model previously adopted and tested in the determination of the gravity fields of Titan (14) and Enceladus (15), implemented in the Jet Propulsion Laboratory (JPL) navigation code MONTE (16). The model was extended to include Saturn's gravitational parameter $G M$, the zonal harmonic coefficients $J_{2^{-}} J_{20}$, the tesseral (longitude-dependent) field of degree 2 (to account for possible non-principal axis rotation), and the mass of the rings. The truncation of the zonal field was set to twice the degree of the highest gravity harmonic whose central value is above the uncertainty (degree 10). The estimate of the even zonal harmonics used in our interpretation (degree $\leq 10)$ is insensitive to changes in the truncation. Although this model was adequate for fitting Juno gravity data at Jupiter (5), obtained in an orbital configuration similar to Cassini's Grand Finale, it could not reduce Cassini's Doppler residuals to the noise level. Instead, small stochastic accelerations were added to the model (see below) to produce signature-free residuals (Fig. S1).

Although all the rings are included in the dynamical model, only rings $\mathrm{A}, \mathrm{B}$ and $\mathrm{C}$ can produce an acceleration potentially detectable by Cassini. The rings are assumed to be coplanar with Saturn's equator and each is assumed to have a constant surface mass density (the data are insensitive to radial variations of the density). Space- and ground-based measurements of ring occultations provide the determination of Saturn's spin axis position and precession rate (17). Although Doppler data are sensitive to the orientation of Saturn's equatorial plane, we adopt the prior determination because it is about ten times more accurate than that obtained from our orbital fitting.

Accelerations that are known to be large enough to produce noticeable signatures on range-rate data were accounted for. These include the point-mass gravitational accelerations from Saturn 
and its satellites (including the ring moons), computed from the JPL planetary and satellite ephemerides DE430, SAT389 and SAT393 (18), the acceleration from the Sun, the planets and satellites of the Solar System, and Saturn's tidal response to its satellites (12).

The acquired Doppler data were combined in a multi-arc, weighted least-squares estimation filter. In the multi-arc approach, the entire time span of the observations is decomposed into shorter intervals and a distinction is made between global and local estimated parameters. Global parameters are common to all arcs and estimated using all available observables. These include Saturn's $G M, J_{2}$ through $J_{20}, C_{21}, C_{22}, S_{21}, S_{22}$, and the masses of the A, B and C rings. Although the estimates of the masses of the three rings are highly correlated, their sum is well determined. The ring masses were initially set to the current best estimates of their values from ring occultation data (19-21), with an a priori uncertainty of $100 \%$ (see Table S2). The mass of the B ring had a large uncertainty of 10 Mimas masses (Saturn's moon Mimas $G M$ is $2.5026 \mathrm{~km}^{3} \cdot \mathrm{s}^{-2}$ ). The sum of the masses of Saturn and its rings was constrained to be equal to the value (and uncertainty) estimated from satellite ephemerides (18).

Local parameters are those that belong only to a single arc and a different value was estimated for each arc. These included the Cassini position and velocity when the gravity observations began in each of the five orbits, at least 12 hours before transit at pericenter. The a priori uncertainties for position and velocity were set at $100 \mathrm{~km}$ and $1 \mathrm{~m} \cdot \mathrm{s}^{-1}$, respectively.

In the estimation filter, parameters whose uncertainties are large enough to contribute to the final covariance matrix have been included as consider parameters. These include the Love number $k_{22}$ (determining Saturn's tidal response), Saturn's pole direction, accelerations due to thermal emission from Cassini's radioisotope thermoelectric generators (RTG), and solar radiation pressure. The nominal value and a priori uncertainty of the Love number are taken from previous Cassini constraints (22), as were the pole position and precession rate (17). The anisotropic thermal acceleration from the RTGs was determined to $5 \%$ or better during the Cassini mission by the Navigation Team. The relative uncertainty associated with solar radiation pressure acceleration was set to $20 \%$.

\section{Gravity determination}

Our deterministic model, based on the geophysical expectations for the gravity field of a gas giant like Saturn, can adequately fit the Doppler data if each arc is analysed separately. However, the same model cannot jointly fit all passes in a combined, multi-arc, gravity and orbital solution. The signatures in range-rate residuals are as large as $0.2 \mathrm{~mm} \cdot \mathrm{s}^{-1}$ over time scales of 20-60 minutes, corresponding to radial accelerations of the order of $10^{-7} \mathrm{~m} \cdot \mathrm{s}^{-2}$. This value is an underestimate of the real unmodeled accelerations, as a large fraction of them are aliased in those associated with estimated parameters. The unmodeled accelerations acting on Cassini must be compensated for, to avoid biases in the estimates of the gravity harmonics and ring mass.

The missing accelerations could be due to longitudinally-varying density anomalies resulting from wind dynamics or convection in Saturn's deep interior (23). For a rocky planet, the corresponding gravitational field would be static and described by tesseral harmonics. However, this approach is not immediately applicable to a fluid planet like Saturn, because vortices move longitudinally with different speeds depending on latitude. The resulting gravity disturbances (caused by the non-zonal wind dynamics) would not be static in any reference frame over the 60- 
day duration of the Cassini gravity measurements. However, if the density anomalies are deepseated, below the level of the zonal winds and in the region of uniform rotation, then a static tesseral field associated with convection might be maintained over time scales much longer than the duration of the experiment.

Although a high-degree tesseral field can fit the data, the required degree increases with the number of passes analysed and depends on the assumed rotation period. Several measurements of Saturn's rotation period have been made using different techniques. In our analysis we use the four values: $10 \mathrm{~h} 32 \mathrm{~m} 45 \mathrm{~s}(24), 10 \mathrm{~h} 39 \mathrm{~m} 22 \mathrm{~s}$, also called System III (25), 10h45m45s (26) and $10 \mathrm{~h} 47 \mathrm{~m} 06 \mathrm{~s}(27)$, leading to a required tesseral field of degree and order ranging from $8 \times 8$ to $12 \times 12$ (Fig S4). However, the determination of the tesseral field amplitudes is non-unique, due to the uncertainty in the underlying rotation rate of the deep interior (12).

Another potential solution is to assume a time-varying field, generated by acoustic oscillations in the planet (28). Fundamental mode oscillations (f-mode, with radial order equal to zero) have been detected by analysis of density waves in the $\mathrm{C}$ ring (29). These waves were also detected in Cassini ring stellar occultation data and are identified with sectoral modes which have an azimuthal order $2 \leq m \leq 10$ (sectoral harmonics depend only on longitude). However, their absolute amplitudes are not well determined. In principle, an infinite number of modes can exist within Saturn, only a subset of which can drive waves in the rings. The inclusion of such normal modes in the dynamical model is possible, but the choice of the relevant modes is not unique. We found that different combinations of modes can fit the data, but that it is impossible to identify unambiguously the dominant ones with the limited data available.

A more general approach, often adopted in orbit determinations in the presence of unknown or poorly-modelled dynamics, is to assume empirical, random accelerations unrelated to any specific physical model. In the absence of geophysical evidence to pinpoint the root cause of the residual accelerations, we used this approach to obtain the baseline solution shown in Table 1. Our goal in this analysis was to disentangle the estimates of the zonal harmonics and the ring mass from the unmodeled accelerations. As an additional check on the robustness of the solution, we compared the baseline solution with solutions obtained using a tesseral field and multiple normal modes (12).

These random accelerations are assumed to act on Cassini's orbit for a limited time span about pericenter and estimated in the orbital frame as local parameters. On each arc, these accelerations can act for equal-duration intervals at a constant value. The duration and the a priori uncertainty of the constant accelerations are adjustable parameters. In principle, it is desirable to optimize the number and duration of the intervals to avoid a degradation of the solution accuracy due to the over-parametrization of the problem. The a priori uncertainty must also be minimized to avoid aliasing part of the zonal gravity signal into the piecewise-constant accelerations. The minimum value of the a priori uncertainty (corresponding approximately to the largest allowed value of the random accelerations) represents the order of magnitude of the dynamical model's incompleteness.

We found that random accelerations with an a priori uncertainty of $4 \times 10^{-7} \mathrm{~m} \cdot \mathrm{s}^{-2}$, acting for 10 minute intervals within \pm 1 hour from pericenter are able to remove the excess signatures in the Doppler residuals. The profile of the estimated random accelerations is reported in Fig. S3.

The reference multi-arc solution is reported in Table 1 and Fig. 1. Prior to Cassini's Grand Finale, the determination of Saturn's even zonal coefficients $J_{2}, J_{4}, J_{6}$ was obtained from 
perturbations of the orbits of the moons, as well as direct perturbations on Cassini itself (18). The data acquired during the Grand Finale orbits are consistent with these previous estimates; they also provide determination of the higher-degree gravity harmonics $\left(J_{8}\right.$ and $\left.J_{10}\right)$ and the odd harmonic coefficients $J_{3}$ and $J_{5}$, the only odd harmonics whose values are larger than the associated uncertainties.

The data effectively constrain the sum of the masses of the A, B and C rings, but the individual masses are poorly determined due to their large mutual correlations. However, the masses of the more-transparent $\mathrm{A}$ and $\mathrm{C}$ rings have been estimated from density waves seen in stellar occultations $(20,21)$. We therefore estimated the masses of the A and C rings subject to those constraints (a priori values and uncertainties). Our final estimates of the $\mathrm{A}$ and $\mathrm{C}$ ring masses are essentially the same as the a priori values (see Table S2). The uncertainty in the total ring mass (Table 1) is obtained from the correlation submatrix of the $\mathrm{A}, \mathrm{B}$ and $\mathrm{C}$ ring masses. We find a value of $0.41 \pm 0.13$ Mimas masses $(1 \sigma)$ for the total ring mass (Table S2).

The alternative tesseral field and normal modes approaches were compared with the piecewiseconstant acceleration case to check the stability of our solution. The three solutions are in agreement with each other, as shown by the error ellipses in Fig. S4 for pairs of gravity harmonics and in Table S2 for the ring masses. We conclude that the estimate of the zonal harmonics is robust and largely independent of the dynamical model. This is especially true for the ring mass, which is consistent in all models and insensitive to the assumptions.

\section{Saturn interior models with uniform rotation}

Although gravity measurements provide constraints on the interior of gas giants, every model using gravity data unavoidably suffers from uncertainties. We tested whether interior models based on uniform rotation can explain the measured gravity harmonics. We developed a suite of interior models based on the common assumption that the fluid in Saturn's interior rotates uniformly like a solid body. All our models have a molecular and a metallic layer, each represented by an adiabat (a constant entropy curve in the temperature-pressure diagram) consistent with an equation of state (EOS) for hydrogen-helium mixtures determined from firstprinciples simulations $(30,31)$, and characterized by an entropy, $\mathrm{S}$, a helium mass fraction, $Y$, and a mass fraction of heavy elements, $Z$. We assume that helium rain occurs in Saturn's interior wherever hydrogen and helium become immiscible, because hydrogen becomes metallic while helium does not (32). We thus introduce a helium rain layer that starts and ends at pressuretemperature conditions that are compatible with the hydrogen-helium immiscibility zone. We treat the helium rain layer as a smooth transition from the parameters across a range of pressures $P_{1}$ to $P_{2}$, defined by the intersections of the adiabat with the immiscibility curve (32). These adiabatic profiles are thus described by six parameters $\left(S_{m o l}, Y_{m o l}, Z_{m o l}, S_{m e t}, Y_{m e t}, Z_{m e t}\right)$, where the subscript 'mol' denotes the outer, molecular envelope and 'met' the inner, metallic envelope. The models match the planet's total mass and include dense cores of various sizes. Initially, we assumed a fractional radius of the core as $r_{c}=0.2$ (33) and later refined the core radii by assuming a terrestrial iron-silicate composition (0.325:0.675) as well as a solar iron-silicatewater ice composition (0.1625:0.3375:0.5), which is consistent with Callisto's interior (34). For these two compositions, we derived fractional core radii of $r_{c}=0.188$ and 0.231 respectively, while assuming hydrostatic equilibrium inside the core and previously-published equations of state (35). 
For each set of parameters, we construct a model for Saturn's interior by performing a calculation with the concentric MacLaurin spheroid (CMS) method $(34,35)$ to find a selfconsistent shape and gravitational field for the planet. The simulation suite includes models with the four uniform rotation periods for Saturn's interior (24-27) observations. A range of interior distributions of helium were adopted, with a gradual gradient at a depth consistent with the phase diagram (32), and the planet-wide mass fraction of helium matching the solar fraction (38). A wide range of $Y_{m o l}$ was considered, bounded by the solar helium fraction $Y=0.274$ and the lowest prediction $Y=0.055$ from infrared measurements of Saturn's atmosphere by Voyager (39). The range in entropy of the deep interior $\left(S_{m e t}\right)$ is between the value in the outer envelope $S_{m o l}=6.84$ and the maximum value consistent with the predicted hydrogen-helium phase separation conditions $S \sim 7.20$. Values of $Z_{m o l}$ up to 5 times solar fraction and fractional core radii up to 0.3 were considered. In each model, the core mass and deep heavy element fraction $\left(Z_{m e t}\right)$ were tuned to match the observed $J_{2}$, and an iterative approach was used to identify the narrower range of model parameters matching the observed $J_{4}$.

The resulting gravity harmonics are compared in Table 2 with the Cassini observations. While a subset of the uniform rotation models is found to simultaneously match the observed $J_{2}$ and $J_{4}$, the magnitude of the observed values for $J_{6}, J_{8}$, and $J_{10}$ are much larger than predicted by the uniform rotation models (Fig. 1 and SM6). Even considering the wide range of physical parameters and interior structures considered, the tight correlation between $J_{4}$ and the higher order harmonics $J_{6^{-}} J_{10}$ precludes any possibility of finding a model that simultaneously matches all the observed harmonics. This result is unlike the gravity measurements of Jupiter from the Juno spacecraft. In this case, static models can be constructed, for which all even harmonics differ by less than $0.1 \times 10^{-6}$ from the measurements $(8)$, while Table 2 shows that the deviations for Saturn are much larger.

To test the robustness of this result, we added additional flexibility to the interior density distribution. Starting from a reasonable physical model, we introduced arbitrary density jumps of up to $\pm 4 \%$ at four points in pressure that were chosen at random. We fit the models to the observed $J_{2}$ value but report the full range of the higher $J_{2 k}$ value without minimizing the discrepancy from the observations. These additional density modifications increased the range of all $J_{2 k}$ value in our ensemble of models (Table 2), but a sizable discrepancy from the observed $J_{8}$, and $J_{10}$ values remained. Fig. 1 and S6, and Table 2 show the large and systematic deviations between the observations and models that assume uniform rotation. This demonstrates that specific assumptions regarding the EOS, and conditions of helium rain cannot explain the inability of the models to match the higher order harmonics.

The inability to reproduce the unusually large values of $J_{6}, J_{8}$, and $J_{10}$ leads us to conclude that models with uniform rotation are ruled out by the gravity data. Instead, the observations require us to introduce differential rotation (DR) (2) into our models for Saturn's interior. We study its effects with two different techniques. First, we introduce DR on cylinders into the CMS method, which allows us to construct consistent interior models but requires constant rotation rate on cylinders that penetrate all the way through the planet. Second, we use the thermal wind method (3) to derive the difference in the gravity signature between a uniformly and a differentially rotating body. The latter approach has the advantage that the DR profiles can have a finite depth and are not required to be north-south symmetric. 


\section{Differential rotation on cylinders with the CMS method}

In the CMS with DR approach we simultaneously optimize interior parameters and DR profiles, $\omega(l)$ where $\omega$ is angular frequency and $l$ is the cylindrical radius from the axis of rotation. We

derive a centrifugal potential, $Q(l)=\int_{0}^{l} d l^{\prime} l^{\prime} \omega\left(l^{\prime}\right)^{2}$, that we introduce into the CMS method $(2,40,41)$. Since this approach is based on potential theory, $\omega(l)$ can only depend on $l$ and may not decay with depth along the cylinders. The benefit of the combined CMS+DR approach is that it represents a fully self-consistent velocity profile, interior density distribution and gravitational field, rather than treating the winds as a correction to a model with uniform rotation.

We find that the assumption of DR can account for the unusually large magnitude of coefficients $J_{6-} J_{10}$. By assuming Saturn's equatorial region rotates approximately $4 \%$ faster than the deep interior, agreement between models and data for all coefficients $J_{2}-J_{10}$ can be achieved (Table 2). This assumption is also compatible with the equatorial part of the wind profile obtained from optical observations (42), showing strong eastward flow near the equator. We thus favored models that were in general agreement with the observed winds when we subsequently constructed an ensemble of interior models. For the planet's deep interior, we assumed the same four different rotation periods as above (24-27).

Starting from parameters set chosen at random, we performed $\sim 10^{4}$ independent model optimizations with the simplex algorithm. The 11 best models were used as starting points for subsequent Markov chain Monte Carlo (MCMC) calculations to explore the allowed parameter space more carefully. Models that matched the observed gravitational moments $J_{2}-J_{10}$ are compared in figures 2 and 3. Fig. 2 shows the rotational velocity profiles as a function of $l$ and compares these to the corresponding north-south averaged surface winds derived from optical tracking of clouds $(42,43)$.

From the best-fitting MCMC models we selected quantities to elucidate the allowed region of parameter space (Fig. 3). Models that assume core masses between 15.0 and 18.2 Earth masses best match the gravity measurements (Fig. 3a). This range of core masses is broadly consistent with predictions of earlier models (44-47) built on previous gravity measurements and different assumptions for the equation of state or structure of the deep interior. The predicted core masses increase if a smaller core radius is assumed because the H-He mixture surrounding the core is exposed to higher pressures and becomes denser. The core masses also increase when a longer rotation period is assumed. As the total planet mass is constrained, the core mass anticorrelates with the fraction of heavy elements in the envelope $\left(Z_{m o l}\right)$. In comparison to the core (which we assume here consists entirely of elements heavier than helium), the mass of heavy elements in the envelope is relatively small, with a permitted range of 1.3 to 4.8 Earth masses. By contrast, analogous interior models of Jupiter predict a core mass slightly lower than Saturn's, but with greater heavy element enrichment in the deep envelope (8). Fig. $3 \mathrm{~b}$ shows the correlation between $Y_{m e t}+Z_{m e t}$ and entropy of the metallic region, $S_{m e t}$. An increase in entropy implies a higher temperature and a lower density. This density reduction can then be compensated by higher fractions of helium and heavy elements.

The helium fraction in the metallic layer and in the molecular layer are linearly related (Fig. 3c), as we require all models to have an overall solar helium abundance in the envelope. All models assumed that some helium rain has occurred, depleting the molecular layer and enriching the 
metallic layer in helium. We find that almost no helium sequestration is predicted for models that assume the longer rotation periods of $10 \mathrm{~h} 45 \mathrm{~m} 45 \mathrm{~s}$ or $10 \mathrm{~h} 47 \mathrm{~m} 06 \mathrm{~s}$. This effectively removes one degree of freedom for the interior models and further constrains the range of other model parameters. For example, when we performed MCMC simulations with variable core radii for a period of $10 \mathrm{~h} 47 \mathrm{~m} 06 \mathrm{~s}$, no models with core radius larger than 0.21 and core masses larger than 17.2 Earth masses were obtained. Fig. 3d shows that the heavy element fraction in the molecular envelope may vary between 1 and 3.5 times the solar value. The heavy $Z$ fraction is anticorrelated with the helium fraction, which implies that there is only limited capacity for elements heavier than hydrogen. Longer rotation periods imply there is a greater capacity for such elements.

All models that match the even gravity harmonics, $J_{2}-J_{10}$, show a rapid decrease in angular velocity from $l=1.0$ to $l \sim 0.90$ (Fig. 2 ). For all rotation periods under consideration, our CMS+DR models also require a minimum region near $l \sim 0.83$ where the fluid rotates slower than in the deep interior. This radius corresponds to a depth of $\sim 10,000 \mathrm{~km}$ from the surface at the equator. By construction, the DR profiles converge to the assumed rotation rate for small $l$. The CMS+DR models are unable to reproduce the wind profile for small $l$ (high latitudes) because we assume DR on cylinders.

\section{Differential rotation with finite depth flows}

An alternative approach, the thermal-wind method (3), can incorporate wind profiles with a finite depth. In this approach, the flow is not limited to full cylindrical symmetry and the flows are not required to be equal in the northern and southern hemispheres. This allows the model to account for both the even and odd gravity harmonics; the latter reflect a north-south asymmetry in the gravity field (4). It has been shown that for the barotropic case (flows limited to cylindrical symmetry), the thermal-wind and the CMS method with DR produce consistent results $(41,48)$. We apply the thermal-wind method using an adjoint inverse model (49), where the measured gravity harmonics in Table 1 are used to identify the flow structure that best fits the data. Although it has these advantages, the thermal-wind method can only account for the dynamical part of the gravity spectrum (due to the flows), and therefore relies on having prior knowledge of a reference interior model for the even gravity harmonics. The predicted gravity values hence depend on the reference model (Table 2).

For Jupiter, the extension of the cloud-level flow into the interior results in a gravity spectrum that matches the observations ( 6 ) and the background density profile. The case of Saturn is more complicated. We find that regardless of the vertical structure of the zonal flow, with the background density profiles corresponding to the 11 models discussed in the previous section, extending the observed cloud-level flow (43) into the interior results in dynamical gravity harmonics $\left(\Delta J_{n}\right)$ that are at least a factor of two too small. For example, the gravity signature of the observed surface winds extended along cylinders to large depths gives roughly $\Delta J_{8}=-1.5 \times 10^{-6}$ and $\Delta J_{10}=1 \times 10^{-6}(4,50)$. The measured $J_{8}$ and $J_{10}$ are $-14.6 \times 10^{-6}$ and $4.7 \times 10^{-6}$, respectively, while the uniform rotation contribution is at most $-9 \times 10^{-6}$ and $1 \times 10^{-6}$ (Table 2). This implies that the observed cloud-level flow cannot account for the difference between the measurements and the uniform rotation contribution regardless of how it extends into the interior. As seen in Fig. 2, the only way to match the data is to alter the latitudinal profile of the zonal wind, by assuming that, 
due to other processes, the bulk of the sub-cloud level flow does not resemble that observed at cloud-level.

Therefore, in order to match the Cassini gravity measurements, another degree of freedom is added to the thermal-wind gravity inversion, whereby the meridional profile of the flow is allowed to vary from the observed winds in addition to the flow depth. We then optimize for the depth and meridional structure of the zonal flow so that the calculated gravity harmonics match the measured values. To compare the results to those of the hemispherically symmetric CMS method, and because unlike Jupiter ( 6 ) the odd harmonics are small and contribute little to the flow structure, we use only the even gravity harmonics, focusing on those which are most strongly affected by the flows, namely $J_{8}$ and $J_{10}$. Due to the uncertainty resulting from the uniform rotation reference model, and the non-uniqueness of the problem, there is little merit in optimizing for the vertical profile of the flow, as can be done for Jupiter ( 6 ), so the vertical decay is approximated by a radial hyperbolic tangent profile with a width of $500 \mathrm{~km}$, and then optimized for its depth.

The reconstructed meridional profile of the zonal flow at the planet's surface is shown in Fig. 4a, achieved with a vertical profile depth of $9363 \pm 357 \mathrm{~km}$. With these parameters, we were able to match the measured values of $J_{8}$ and $J_{10}$, once the uniform rotation contribution in Table 2 is subtracted. Both gravity harmonics are within the uncertainties of the measured values (Table 3 ). The $\Delta J$ we optimize for are calculated from the difference between the measurements and the 11 preferred models run without DR and using the Voyager rotation period. Although the reconstructed zonal wind profile varies from the observed one, it stills retains its main characteristics (Fig. 4a). The uncertainty of the wind depth is obtained from the variance in the 11 solutions of the internal models, the variance in the internal models and thermal-wind calculation for different rotation rates between $10 \mathrm{~h} 32 \mathrm{~m}$ and $10 \mathrm{~h} 47 \mathrm{~m}$, and using different internal models, by statistically varying the depth, and finally searching for solutions that satisfy the condition that both harmonics are within the uncertainties. In all cases the reported depth is the inflection point of the hyperbolic tangent vertical profile. The exact meridional structure of the zonal winds is not uniquely determined, meaning that other profiles can give results within the measurement uncertainties.

Regardless of the exact meridional profile, all vertical flow profiles are constrained to contain a very deep flow of about $9000 \mathrm{~km}$. This depth, corresponding to $15 \%$ of Saturn's radius, matches the predictions from magnetohydrodynamic theories, suggesting that the flow should extend down to the levels of magnetic dissipation $(51,52)$. It has been suggested that a higher order expansion to the vorticity balance, beyond a thermal wind, is necessary to reproduce the dynamical gravity harmonics (53). To compare those solutions (known as the thermal-gravity equation, 53) we calculate the gravity harmonics using the thermal-gravity equation for a similar wind profile. The comparison (Table 3 ) shows that the solutions from both methods are within the observational uncertainties, confirming that the thermal wind solution is the leading order vorticity balance (48). The thermal-gravity solution was obtained by solving the full integrodifferential equation on a sphere (54).

The CMS solutions with DR on full cylinders show a flow signature only for distances from the axis of rotation $l \geq 0.6$ (Fig. 2). Using the thermal-wind inversion model, we can examine the nature of the optimized flow obtained under a similar restriction. In Fig. $4 \mathrm{~b}$ we show that taking the same approach with the thermal-wind model, restricting the flow to be equatorward only for 
$l \geq 0.6$ (latitudes $\leq 60^{\circ}$ ), gives similar results. The reconstructed flow in this case is not as close to the observed flow as in the unrestricted case (Fig. 4a), but still gives results within $1 \sigma$ of the deviation uncertainty (Table 3). We find a large flow depth of $8832 \pm 295 \mathrm{~km}$ also in this solution. The deviations between the two flow models in Fig. 4 illustrate the non-uniqueness in the resulting flows when gravity harmonics $\Delta J_{8}$ and $\Delta J_{10}$ are modeled. However, all our thermalwind flow solutions show strong westward flow near latitude $35^{\circ}$, which is in agreement with the westward flowing region calculated with the CMS model in Fig. 2. These results were calculated for a rotation period of $10 \mathrm{~h} 39 \mathrm{~m} 22 \mathrm{~s}$; because the high-degree harmonics are weakly affected by the rotation rate (55), we expect consistent results if a different period is assumed.

We find therefore that with the thermal wind approach, extending the exact observed flows into the interior in a simple manner does not lead to an exact match of measured gravity values. However, flow profiles that are similar in general character to the observed ones (Fig. 4) yield solutions within the deviation uncertainty when extended to a depth of $\sim 9000 \mathrm{~km}$, indicating that the flows are very deep and likely extend down to the levels of magnetic dissipation.

The agreement between the CMS and thermal wind solutions, based on two substantially different approaches, indicate that the interpretation of the Cassini gravity data is robust. Regardless of the exact interior model or vertical decay profile chosen, the flows must extend deep below the surface, to about $15 \%$ of the planet's radius.

\section{Mass and age of Saturn's rings}

We now consider the mass of the main rings (i.e., the $\mathrm{A}, \mathrm{B}$ and $\mathrm{C}$ rings) and how this quantity can constrain theoretical models for the age and origin of the rings. (The masses of the diffuse D, F, G and E rings are expected to be negligible.) Prior to the Voyager flybys in $1980 / 81$, it was known that the cross-section-weighted average ring particle radius was at least $10 \mathrm{~cm}$, as required to account for the rings' high radar reflectivity (56). Voyager results provided estimates of the rings' mass, based on local surface mass densities determined from the wavelengths of thirteen density waves in the A and B rings and an optical depth profile derived from a stellar occultation (57). The estimated total ring mass was $2.8 \times 10^{19} \mathrm{~kg}$, or 0.75 Mimas masses.

However, it was subsequently argued that substantially more mass might be hidden in the opaque parts of the B ring (58).

Cassini observations of density waves in the rings have led to additional local surface density estimates of the A ring (20), C ring (29), and the B ring (19). The B ring density is higher than the others, with a mean value of $\sim 600 \mathrm{~kg} \cdot \mathrm{m}^{-2}$, but not as much higher as its large optical depth would suggest. Combining these estimates, we find a likely total ring mass of $G M=1.01 \mathrm{~km}^{3} \cdot \mathrm{s}^{-2}$ or 0.40 Mimas masses. However, the presence of self-gravity wakes in the A and B ring has led to suggestions that substantial amounts of material may be present but does not contribute to density wave estimates. Numerical simulations of these structures (58) suggest wake surface densities as high as $5000 \mathrm{~kg} \cdot \mathrm{m}^{-2}$ and an upper limit to the total ring mass of $9.7 \times 10^{19} \mathrm{~kg}$, or $\sim 2.5$ Mimas masses.

Our estimate of $G M=0.58 \pm 0.48 \mathrm{~km}^{3} \cdot \mathrm{s}^{-2}$ for the B ring, when combined with the previouslydetermined $G M$ products of the $\mathrm{A}\left(0.38 \mathrm{~km} \cdot \mathrm{s}^{-2}\right)$ and $\mathrm{C}\left(0.06 \mathrm{~km}^{3} \cdot \mathrm{s}^{-2}\right)$ rings, leads to a total ring $G M$ of $1.02 \pm 0.41 \mathrm{~km}^{3} \cdot \mathrm{s}^{-2}$, equivalent to $0.41 \pm 0.13$ Mimas masses (Table S2). This estimate is 
somewhat smaller than the Voyager value, but consistent with those inferred from Cassini observations of density waves.

The ring mass has implications for the age of the rings (59). Traditional age estimates of Saturn's ring-satellite system fall into three categories: dynamical estimates based on the rate of recession of the small satellites from the rings due to gravitational torques, and the back-reaction on the A ring $(10,60)$; structural evolution timescales based on the evolution of unconfined edges such as the inner edges of the $\mathrm{A}$ and $\mathrm{B}$ rings (11); and compositional timescales based on the assumption that the rings were formed as pure water ice and have subsequently been steadily darkened by the infall of interplanetary debris (9). Most of these timescale estimates depend, directly or indirectly, on the mass of the rings; both dynamical and compositional considerations suggest that low-mass rings are likely to be young and both approaches yield evolutionary ages $\sim 10^{8}$ yrs for the A and B rings, assuming the Voyager measurements of ring masses and interplanetary impact fluxes (59). Low-mass rings therefore pose a challenge for models in which the ring system is assumed to have formed in the early Solar System.

The Cassini results have sharpened these arguments. Analysis of the main rings' non-icy fraction derived from their passive microwave emission, using the pre-Cassini interplanetary flux estimates and a minimum-mass $\mathrm{B}$ ring, led to estimates of the ages of the A and B rings of 80$150 \mathrm{Myr}$ and 30-100 Myr, respectively (61). Our revised mass estimate for the B ring would increase the latter estimate by $\sim 25 \%$. Cassini dust measurements have also provided a refined estimate of the interplanetary dust flux at the rings (62) indicating that the interplanetary dust impact flux on the rings is higher by almost a factor of 10, compared to the Voyager estimates, due to improved understanding of the gravitational focusing by Saturn. These new dust fluxes are would reduce the age estimates for the $\mathrm{A}$ and $\mathrm{B}$ rings.

As the rings evolve viscously, they may spread radially beyond the Roche limit and therefore gradually lose mass to form new generations of small satellites, which would then move rapidly away from the rings (63). In this scenario, the rings would have been more massive in the past, with proportionally longer evolutionary timescales. Current models of viscous evolution (64) predict that such a ring would approach an asymptotic mass of $\sim 1.5 \times 10^{19} \mathrm{~kg}$ or 0.40 Mimas masses after $5 \mathrm{Gyr}$, close to our estimate of $0.41 \pm 0.13$ Mimas masses. Taken at face value, and subject to the limitations of the simulations (which ignore the gravitational torques from the newly-formed moons), this suggests that the rings may be older and formed with more mass than they have today. However, the simulations also show that a massive, primordial ring initially loses mass very quickly before settling into a long period of progressively slower evolution (64). An old high-mass ring would thus quickly evolve to a mass not much greater than that measured today, and would therefore still be subjected to rapid ballistic and compositional evolution.

On balance, we favor a scenario whereby the present rings of Saturn are relatively young, at least compared to the planet itself, although they may have evolved substantially in the past $10^{7}-10^{8}$ yrs and were perhaps once more massive than they are today. Our data do not indicate how the ring system formed within such a recent period. Models of that process invoke the chance capture and tidal disruption of a comet or Centaur $(65,66)$. Alternatively, rings may arise from the catastrophic disruption of an earlier population of mid-sized icy satellites $100 \mathrm{Myr}$ ago (67), although whether the resulting debris can migrate into the ring region before it re-accretes into new satellites is uncertain (68). Regardless of how the rings formed, the ring mass we derived from Cassini gravity data indicates a recent origin for Saturn's ring system, which we consider a fitting way to end Cassini's mission. 


\section{References and notes:}

1. W. B. Hubbard, Planetary interiors (Van Nostrand Reinhold, 1984).

2. W. B. Hubbard, NOTE: Gravitational signature of Jupiter's deep zonal flows. Icarus 137, 357-359 (1999).

3. Y. Kaspi, W. B. Hubbard, A. P. Showman, G. R. Flierl, Gravitational signature of Jupiter's internal dynamics. Geophysical Research Letters 37 (2010).

4. Y. Kaspi, Inferring the depth of the zonal jets on Jupiter and Saturn from odd gravity harmonics. Geophysical Research Letters 40, 676-680 (2013).

5. L. Iess et al., The measurement of Jupiter's asymmetric gravity field. Nature 555, 220222 (2018).

6. Y. Kaspi et al., Jupiter's atmospheric jet-streams extend thousands of kilometers deep. Nature 555, 223-226 (2018).

7. T. Guillot et al., A suppression of differential rotation in Jupiter's deep interior. Nature 555, 227-230 (2018).

8. S. M. Wahl et al., Comparing Jupiter interior structure models to Juno gravity measurements and the role of a dilute core. Geophysical Research Letters 44, 4649-4659 (2017).

9. J. N. Cuzzi, P. R. Estrada, Compositional evolution of Saturn's rings due to meteoroid bombardment. Icarus 132, 1-35 (1998).

10. N. Borderies, P. Goldreich, S. Tremaine, "Unsolved problems in planetary ring dynamics" in Planetary rings (Univ of Arizona Press, 1984), pp. 713-734.

11. P. R. Estrada, R. H. Durisen, J. N. Cuzzi, D. A. Morgan, Combined structural and compositional evolution of planetary rings due to micrometeroid impacts and ballistic transport. Icarus 252, 415-439 (2015).

12. Materials and methods are provided in the supplementary material.

13. L. Iess et al., Astra: Interdisciplinary study on enhancement of the end-to-end accuracy for spacecraft tracking techniques. Acta Astronautica 94, 699-707 (2014).

14. L. Iess et al., The tides of Titan. Science 337, 457-459 (2012).

15. L. Iess et al., The gravity field and interior structure of Enceladus. Science 344, 78-80 (2014).

16. S. Evans et al., MONTE: the next generation of mission design and navigation software. CEAS Space Journal 10, 79-86 (2018).

17. R. G. French et al., Noncircular features in Saturn's rings IV: Absolute radius scale and Saturn's pole direction. Icarus 290, 14-45 (2017).

18. https://naif.jpl.nasa.gov/pub/naif/generic kernels/spk/

19. M. M. Hedman, P.D. Nicholson, The B-ring's surface mass density from hidden density waves: Less than meets the eye?. Icarus 279, 109-124 (2016). 
20. M. S. Tiscareno, J. A. Burns, P. D. Nicholson, M. M. Hedman, C. C. Porco, Cassini imaging of Saturn's rings. II. A wavelet technique for analysis of density waves and other radial structure in the rings. Icarus 189, 14-34 (2007).

21. K. Baillié, J. E. Colwell, J. J. Lissauer, L. W. Esposito, M. Sremčević, Waves in Cassini UVIS stellar occultations. 2. The C ring. Icarus 216, 292-308 (2011).

22. V. Lainey et al., New constraints on Saturn's interior from Cassini astrometric data. Icarus 281, 286-296 (2017).

23. D. Kong, K. Zhang, G. Schubert, Using Jupiter's gravitational field to probe the Jovian convective dynamo. Scientific reports 6 (2016).

24. R. Helled, E. Galanti, Y. Kaspi, Saturn's fast spin determined from its gravitational field and oblateness. Nature 520, 202-204 (2015).

25. M. D. Desch, M. L. Kaiser, Voyager measurement of the rotation period of Saturn's magnetic field. Geophys. Res. Lett. 8, 253-256 (1981).

26. D. A. Gurnett et al., Radio and Plasma Wave Observations at Saturn from Cassini's Approach and First Orbit. Science 307, 1255-1259 (2005).

27. G. Giampieri, M. K. Dougherty, E. J. Smith, C. T. Russell, A regular period for Saturn's magnetic field that may track its internal rotation. Nature 441, 62-64 (2006).

28. D. Durante, T. Guillot, L. Iess, The effect of Jupiter oscillations on Juno gravity measurements. Icarus 282, 174-182 (2017).

29. M. M. Hedman, P. D. Nicholson, More Kronoseismology with Saturn's rings. Monthly Notices of the Royal Astronomical Society 444, 1369-1388 (2014).

30. B. Militzer, Equation of state calculations of hydrogen-helium mixtures in solar and extrasolar giant planets. Phys. Rev. B 97, 014202 (2013).

31. B. Militzer, W. B. Hubbard, Ab initio equation of state for hydrogen-helium mixtures with recalibration of the giant-planet mass-radius relation. The Astrophysical Journal 774, 148 (2013).

32. M. A. Morales, S. Hamel, K. Caspersen, E. Schwegler, Hydrogen-helium demixing from first principles: from diamond anvil cells to planetary interiors. Physical Review B 87, 174105 (2013).

33. S. M. Wahl, W. B. Hubbard, B. Militzer, The Concentric Maclaurin Spheroid method with tides and a rotational enhancement of Saturn's tidal response, Icarus 282, 183 (2017).

34. O.L. Kuskov, V.A. Kronrod, Internal structure of Europa and Callisto. Icarus 177, 550569 (2005).

35. S. Seager, M. Kuchner, C. A. Hier-Majumder, B. Militzer, Mass-radius relationship of solid exoplanets, Astrophys. J. 669, 1279 (2007).

36. W. B. Hubbard, High-precision Maclaurin-based models of rotating liquid planets. Astrophysical Journal Letters 756, L15 (2012). 
37. W. B. Hubbard, Concentric Maclaurin spheroid models of rotating liquid planets. The Astrophysical Journal 768, 43 (2013).

38. M Asplund, et al., The chemical composition of the Sun. Astrophysics and Space Science, 328(1), 179-183 (2010).

39. B. J. Conrath, D. Gautier, R. A. Hanel, J. S. Hornstein, The helium abundance of Saturn from Voyager measurements. The Astrophys. Journal 282, 807-815 (1984).

40. J. Wisdom, W. B. Hubbard, Differential rotation in Jupiter: A comparison of methods. Icarus 267, 315-322 (2016).

41. Y. Kaspi, J. E. Davighi, E. Galanti, W. B. Hubbard, The gravitational signature of internal flows in giant planets: Comparing the thermal wind approach with barotropic potential-surface methods. Icarus 276, 170-181 (2016).

42. A. Sánchez-Lavega, J. F. Rojas, P. V. Sada, Saturn's zonal winds at cloud level. Icarus 147, 405-420 (2000).

43. E. García-Melendo, S. Pérez-Hoyos, A. Sánchez-Lavega, R. Hueso, Saturn's zonal wind profile in 2004-2009 from Cassini ISS images and its long-term variability. Icarus 215, 62-74 (2011).

44. W. B. Hubbard, M. S. Marley, Optimized Jupiter, Saturn, and Uranus Interior Models. Icarus 78, 102 (1989).

45. N. Nettelmann, R. Püstow, R. Redmer, Saturn layered structure and homogeneous evolution models with different EOSs. Icarus 225, 548-557 (2013).

46. D. Saumon, T. Guillot, Shock compression of deuterium and the interiors of Jupiter and Saturn. The Astrophysical Journal 609, 1170 (2004).

47. R. Helled, T. Guillot, Interior models of Saturn: including the uncertainties in shape and rotation. The Astrophysical Journal 767, 113 (2013).

48. E. Galanti, Y. Kaspi, E. Tziperman, A full, self-consistent treatment of thermal wind balance on oblate fluid planets. Journal of Fluid Mechanics 810, 175-195 (2017).

49. E. Galanti, Y. Kaspi, An adjoint based method for the inversion of the Juno and Cassini gravity measurements into wind fields. The Astrophysical Journal 820, 91 (2016).

50. E. Galanti, Y. Kaspi, Decoupling Jupiter's deep and atmospheric flows using the upcoming Juno gravity measurements and a dynamical inverse model. Icarus 286, 46-55 (2017).

51. J. Liu, P. M. Goldreich, D. J. Stevenson, Constraints on deep-seated zonal winds inside Jupiter and Saturn. Icarus 196, 653-664 (2008).

52. H. Cao, D. Stevenson, Zonal flow magnetic field interaction in the semi-conducting region of giant planets, Icarus 296, 59-72 (2017).

53. D. Kong, K. Zhang, G. Schubert, Odd gravitational harmonics of Jupiter: effects of spherical versus nonspherical geometry and mathematical smoothing of the equatorially antisymmetric zonal winds across the equatorial plane. Icarus 277, 416-423 (2016). 
54. K. Zhang, D. Kong, G. Shubert, Thermal-gravitational wind equation for the windinduced gravitational signature of giant gaseous planets: mathematical derivation, numerical method, and illustrative solutions. The Astrophysical Journal 806, 270 (2015)

55. E. Galanti, Y. Kaspi, Prediction for the flow-induced gravity field of Saturn: implications for Cassini’s Grand Finale. Astrophys. J. Let. 843, L25 (2017).

56. J.N. Cuzzi et al., "Saturn's rings - Properties and processes" in Planetary rings (Univ of Arizona Press, 1984), pp. 73-199.

57. L. W. Esposito, M. O’Callaghan, R. A. West, The structure of Saturn's rings Implications from the Voyager stellar occultation. Icarus 56, 439-452 (1983).

58. S. J. Robbins, G. R. Stewart, M. C. Lewis, J. E. Colwell, M. Sremčevića, Estimating the masses of Saturn's A and B rings from high-optical depth N-Body simulations and stellar occultations. Icarus 206, 431-445 (2010)

59. S. Charnoz, “Origin and evolution of Saturn's ring system” in Saturn from CassiniHuygens, M. Dougherty, L. Esposito, S. Krimigis, Eds. (Springer 2009), chap. 17, pp. 537-575.

60. R. Tajeddine, P.D. Nicholson, P.-Y. Longaretti, M. El Moutamid, J.A. Burns, What Confines the Rings of Saturn?. The Astrophysical Journal Supplement Series 232, 28 (2017).

61. Z. Zhang et al., Exposure age of Saturn's A and B rings, and the Cassini division as suggested by their non-icy material content. Icarus 294, 14-42 (2017).

62. S. Kempf, The age of Saturn's rings constrained by the meteroid flux into the system. Paper presented at the AGU Fall Meeting, New Orleans, LA, 13 December 2017.

63. S. Charnoz, J. Salmon, A. Crida, The recent formation of Saturn's moonlets from viscous spreading of the main rings. Nature 465, 752-754 (2010).

64. J. Salmon, S. Charnoz, A. Crida, A. Brahic, Long-term and large-scale viscous evolution of dense planetary rings. Icarus 209, 771-785 (2010).

65. L. Dones, A recent cometary origin for Saturn's rings?. Icarus 92, 194-203 (1991).

66. H. C. Dones, C. B. Agnor, E. Asphaug, Formation of Saturn's rings by tidal disruption of a centaur. Bulletin of the American Astronomical Society 39, 7.07 (2007).

67. M. Ćuk, L. Dones, D. Nesvorný, Dynamical evidence for a late formation of Saturn's moons. The Astrophysical Journal 820, 97 (2016).

68. R. Hyodo, S. Charnoz, Dynamical evolution of the debris disk after a satellite catastrophic disruption around Saturn. The Astronomical Journal 154, 34 (2017).

69. K. Lodders, H. Palme, H. P. Gail, Solar System (Springer Berlin Heidelberg, Berlin, Heidelberg, 2009), vol. 4B.

70. D. Brouwer, G. Clemence, Methods of Celestial Mechanics (New York Academic press, 1967).

71. J.H. Waite et al., Chemical interactions between Saturn's atmosphere and its rings. Science 362 (2018). 
72. D. Kong, K. Zhang, G. Schubert, Using Jupiter's gravitational field to probe the Jovian convective dynamo. Scientific reports 6, 23497 (2016).

73. T. V. Gudkova, V. N. Zharkov, Theoretical free oscillations spectrum for Saturn interior models. Advances in Space Research 38, 764-769 (2006).

\section{Acknowledgements}

We thank J. W. Armstrong, J. Cuzzi, M. Di Benedetto, S. Finocchiaro, J. Fortney, R. French, L. Gomez-Casajus, M. Gregnanin, M. Hedman, R. A. Jacobson, E. Marouf, N. Movshovitz and L. Spilker for their suggestions and contributions. We are grateful to the Cassini Radio Science operations team members (E. Barbinis, D. Kahan, J. Gao, C. Lee) for supporting the experiments described in this paper. We also thank the DSN and ESA personnel involved in the acquisition of the data. L.I. dedicates this work to the memoir of Bruno Bertotti.

\section{Funding}

Support for this work was provided by the Italian Space Agency (LI, DD, PR, MM, PT, MZ), NASA's CDAP program (BM, WH, SW), by the Cassini Project (PN, AA) and the Israeli Space Agency (YK and EG). All authors acknowledge support from the Cassini Project.

\section{Author contributions}

L.I. led the experiment and supervised the data analysis. D.D. and P.R. carried out the gravity data analysis. B.M., WH and SW contributed interior models and the differential rotation with CMS method. Y.K. and E.G. contributed the models on differential rotation with finite depth flows. P.N. contributed the ring mass interpretation. A.A. coordinated the experiment operations and data acquisition. M.J.M., P.T., and M.Z. helped in the data analysis.

\section{Competing interests:}

The authors declare no competing interests.

\section{Data and code availability}

The Cassini data used in this paper is available through the NASA Planetary Data System at https://atmos.nmsu.edu/pdsd/archive/data/co-s-rss-1-sagr18-v10/ and https://atmos.nmsu.edu/pdsd/archive/data/co-s-rss-1-sagr19-v10/. The MONTE space navigation code was obtained through a license agreement between NASA and the Italian Space Agency; the terms do not permit redistribution. MONTE licenses may be requested at https://montepy.jpl.nasa.gov/. The codes used in the interpretation of gravity data are available on GitHub. Our output gravitational field model is listed in Table 1.

\section{Supplementary Materials}

Materials and Methods

Table S1 - S2 
Fig S1 - S6

References (70-72) 


\section{FIGURES}

Fig. 1: Zonal gravity harmonic coefficients $\boldsymbol{J}_{2}-\boldsymbol{J}_{12}$. The dashed line shows the uncertainties from Table 1. Positive values are marked as solid circles, negative values as empty circles. Theoretical predictions for uniform rotation (Table 2) are plotted with red diamonds (solid for positive values, empty for negative values) and diverge from the measurements at degrees $>8$ (see also Fig. S6 for a comparison with Jupiter).

Fig. 2: Differential rotation profiles for CMS models. CMS profiles are compared with northsouth symmetrized wind profiles from optical observations (42) (black) and (43) (grey). Our Monte Carlo averages for models with three different rotation periods, $P$, and core radius of $r_{c}=0.2$ are plotted.

Fig. 3: Composition of parameters from six sets of interior models including differential rotation. The assumed rotation periods and core radii are indicated by the color and symbol, as specified in the legend. (a) The distribution of heavy element mass between the core and envelope, (b) the variation of the mass fraction of helium and hydrogen plus heavy elements with entropy in the metallic envelope, (c) the variation of helium mass fraction in the molecular and metallic envelopes, (d) the tradeoff between heavy element and helium mass fractions in the molecular envelope. In panels (b) and (c) solar values (69) are shown with a yellow star.

Figure 4: Comparison of observed and reconstructed wind profiles. Observed zonal winds relative to the Voyager rotation rate $10 \mathrm{~h} 39 \mathrm{~m} 32 \mathrm{~s} \mathrm{(43)}$ and meridional profiles of the zonal wind, optimized with the thermal wind method, for (a) a full pole-to-pole wind profile, and (b) a wind profile truncated at latitude $60^{\circ}$. In both cases, the flows must to extend to a depth of $\sim 9000 \mathrm{~km}$ for the computed gravity signal to be consistent with observations for $J_{8}$ and $J_{10}$. The dashed blue line is one of the CMS models shown in Fig. 2 projected into geocentric latitude at the planet's surface, and the red shading is the calculation uncertainty on the optimized meridional profile of the zonal wind. 


\section{TABLES}

Table 1: Measured gravity harmonic coefficients of Saturn (un-normalised; reference radius $60330 \mathrm{~km}$ ) and total ring mass (in units of Mimas' mass). The $J_{2}$ value includes a constant tidal term owing to the average tidal perturbation from the satellites. The associated uncertainties are recommended values intended to be used for analysis and interpretation. For the zonal harmonics they correspond to 3 times the formal uncertainties. The solution for the total ring mass $(A+B+C)$ is stable independently of the adopted dynamical model (Table S2) and the uncertainty reported is the $1 \sigma$ formal uncertainty. See Table S2 for our total ring mass estimates for several models of the unknown accelerations.

\begin{tabular}{|c|c|c|}
\hline & Value & Uncertainty \\
\hline$J_{2}\left(\times 10^{6}\right)$ & 16290.573 & 0.028 \\
\hline$J_{3}\left(\times 10^{6}\right)$ & 0.059 & 0.023 \\
\hline$\left.J_{4}\left(x_{10}\right)^{6}\right)$ & -935.314 & 0.037 \\
\hline$J_{5}\left(\mathrm{x} 0^{6}\right)$ & -0.224 & 0.054 \\
\hline$J_{6}\left(\times 10^{6}\right)$ & 86.340 & 0.087 \\
\hline$J_{7}\left(\mathbf{x} 10^{6}\right)$ & 0.108 & 0.122 \\
\hline$J_{8}\left(\times 10^{6}\right)$ & -14.624 & 0.205 \\
\hline$J_{9}\left(\times 10^{6}\right)$ & 0.369 & 0.260 \\
\hline$J_{10}\left(\times 10^{6}\right)$ & 4.672 & 0.420 \\
\hline$J_{11}\left(\mathrm{x}^{10^{6}}\right)$ & -0.317 & 0.458 \\
\hline$J_{12}\left(\times 10^{6}\right)$ & -0.997 & 0.672 \\
\hline $\operatorname{Ring} \operatorname{mass}\left(M_{M}\right)$ & 0.41 & 0.13 \\
\hline
\end{tabular}


Table 2: Comparison of observed and calculated gravitational harmonics (un-normalised; reference radius $60330 \mathrm{~km}$ ). Where two values are given they denote the minimum and maximum values from the suite of models. The physical models in column 3 match the observed $J_{2}$ and $J_{4}$ in Table 1 , over a parameter space considering ranges of $S_{m e t}, Y_{m o l}, Z_{m o l}, r_{c}$ and rotation periods from $10 \mathrm{~h} 32 \mathrm{~m} 44 \mathrm{~s}$ to $10 \mathrm{~h} 47 \mathrm{~m} 06 \mathrm{~s}$. For the same span of rotation periods, column 4 reports a wider range from models that match only $J_{2}$ and allow for density modifications assuming $r_{c}=0.2$. For $J_{6}-J_{10}$, the discrepancy between measurements and uniform rotation models is large for all models that assume uniform rotation. Column 5 shows a representative model with DR on cylinders and a deep rotation period of $10 \mathrm{~h} 39 \mathrm{~m} 22 \mathrm{~s}$ that matches measurements from $J_{2}$ to $J_{10}$.

\begin{tabular}{|c|c|c|c|c|c|c|}
\hline & Measurements & \multicolumn{2}{|c|}{$\begin{array}{l}\text { Physical models with } \\
\text { uniform rotation }\end{array}$} & \multicolumn{2}{|c|}{$\begin{array}{c}\text { Uniform rotation } \\
\text { model with modified } \\
\text { density profiles }\end{array}$} & $\begin{array}{c}\text { Physical model } \\
\text { with differential } \\
\text { rotation }\end{array}$ \\
\hline$J_{2}$ & $16290.573 \pm 0.028$ & \multicolumn{2}{|c|}{16290.57} & \multicolumn{2}{|c|}{16290.57} & 16290.573 \\
\hline$J_{4}$ & $-935.314 \pm 0.037$ & \multicolumn{2}{|c|}{-935.31} & -990.12 & -902.93 & -935.312 \\
\hline$J_{6}$ & $86.340 \pm 0.087$ & 80.74 & 81.76 & 75.69 & 90.42 & 86.343 \\
\hline$J_{8}$ & $-14.624 \pm 0.205$ & -8.96 & -8.70 & -10.26 & -7.97 & -14.616 \\
\hline$J_{10}$ & $4.672 \pm 0.420$ & 1.08 & 1.13 & 0.97 & 1.33 & 4.677 \\
\hline
\end{tabular}


Table 3: Contribution to the higher gravity harmonics $\Delta J_{8}$ and $\Delta J_{10}$ resulting from differential rotation and thermal-wind optimization. The deviation (Column 1 ) is the difference between the measured $J_{8}$ and $J_{10}$ (Table 1) and the average of the computed values from the 11 CMS models with uniform rotation (Table 2). Two optimizations are shown: one without latitudinal truncation of the zonal flow, resulting in the reconstructed zonal wind profile shown in Fig. 4a and with a flow depth of $9363 \mathrm{~km}$ (Column 2), and the second with the flows truncated at latitude $60^{\circ}$ (Fig. 4b) and a flow depth of $8832 \mathrm{~km}$ (Column 3). Columns 4 and 5 show the deviations calculated with the thermal-gravity equation (48) for similar wind profiles. The solutions from thermal wind are closer to the measurement because the optimization was done using the thermal wind method, but the thermal-gravity solutions also match the observations within $10 \%$.

\begin{tabular}{|c|c|c|c|c|c|}
\hline Deviation & $\begin{array}{c}\text { Thermal-wind } \\
\text { solution }\end{array}$ & $\begin{array}{c}\text { Thermal-wind } \\
\text { solution truncated } \\
\text { at latitude } \mathbf{6 0}^{\circ}\end{array}$ & $\begin{array}{c}\text { Thermal- } \\
\text { gravity } \\
\text { solution }\end{array}$ & $\begin{array}{c}\text { Thermal-gravity } \\
\text { solution truncated } \\
\text { at latitude } \mathbf{6 0}^{\circ}\end{array}$ \\
\hline $\boldsymbol{\Delta J}_{\boldsymbol{s}}$ & $\begin{array}{c}-5.600 \pm \\
0.205\end{array}$ & -5.624 & -5.533 & -5.758 & -5.759 \\
\hline $\boldsymbol{\Delta J}_{\boldsymbol{1 0}}$ & $\begin{array}{c}3.528 \pm \\
0.659\end{array}$ & 3.570 & 3.660 & 3.974 & 4.037 \\
\hline
\end{tabular}




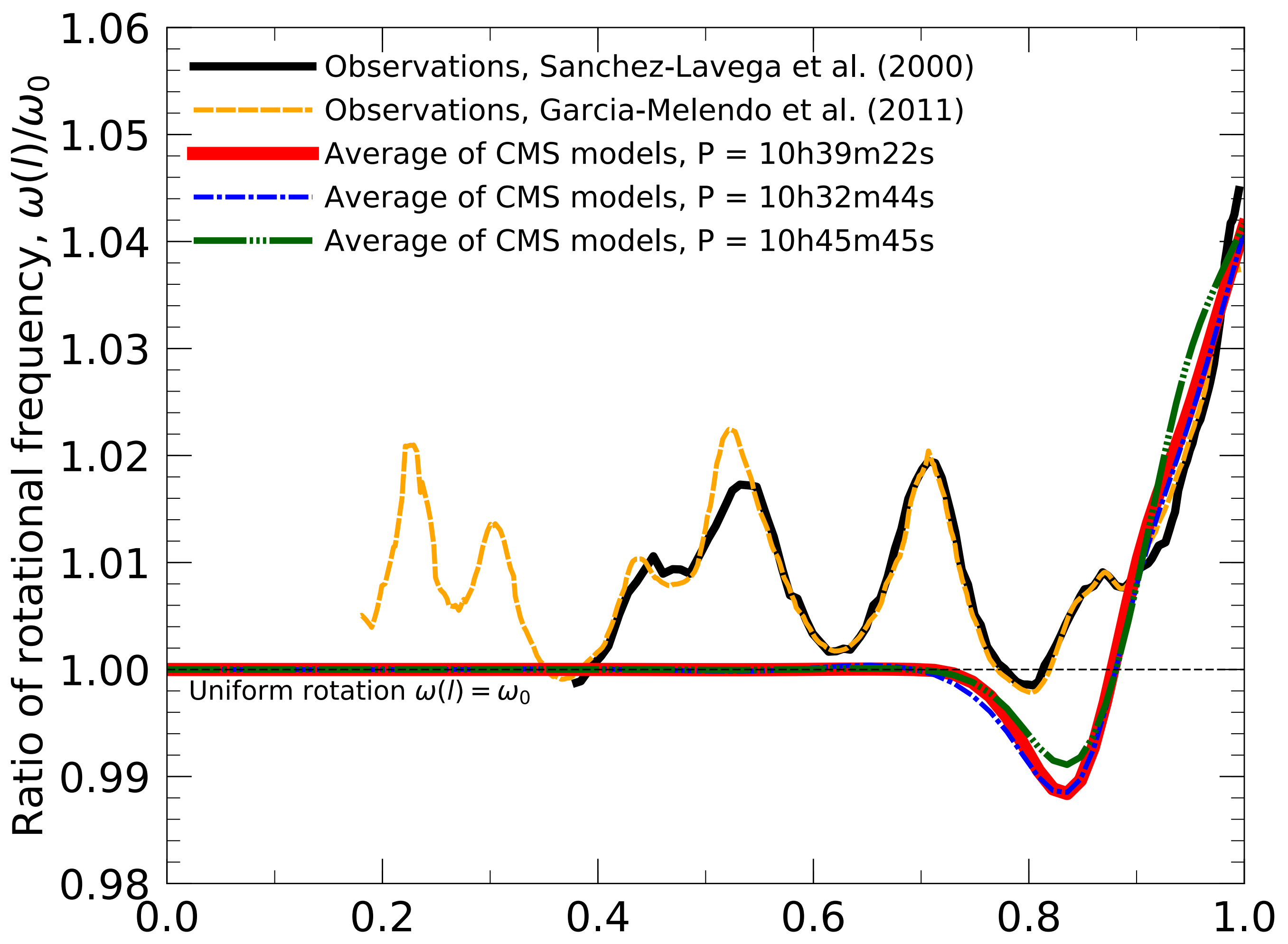
Distance from rotation axis / equatorial radius, I 


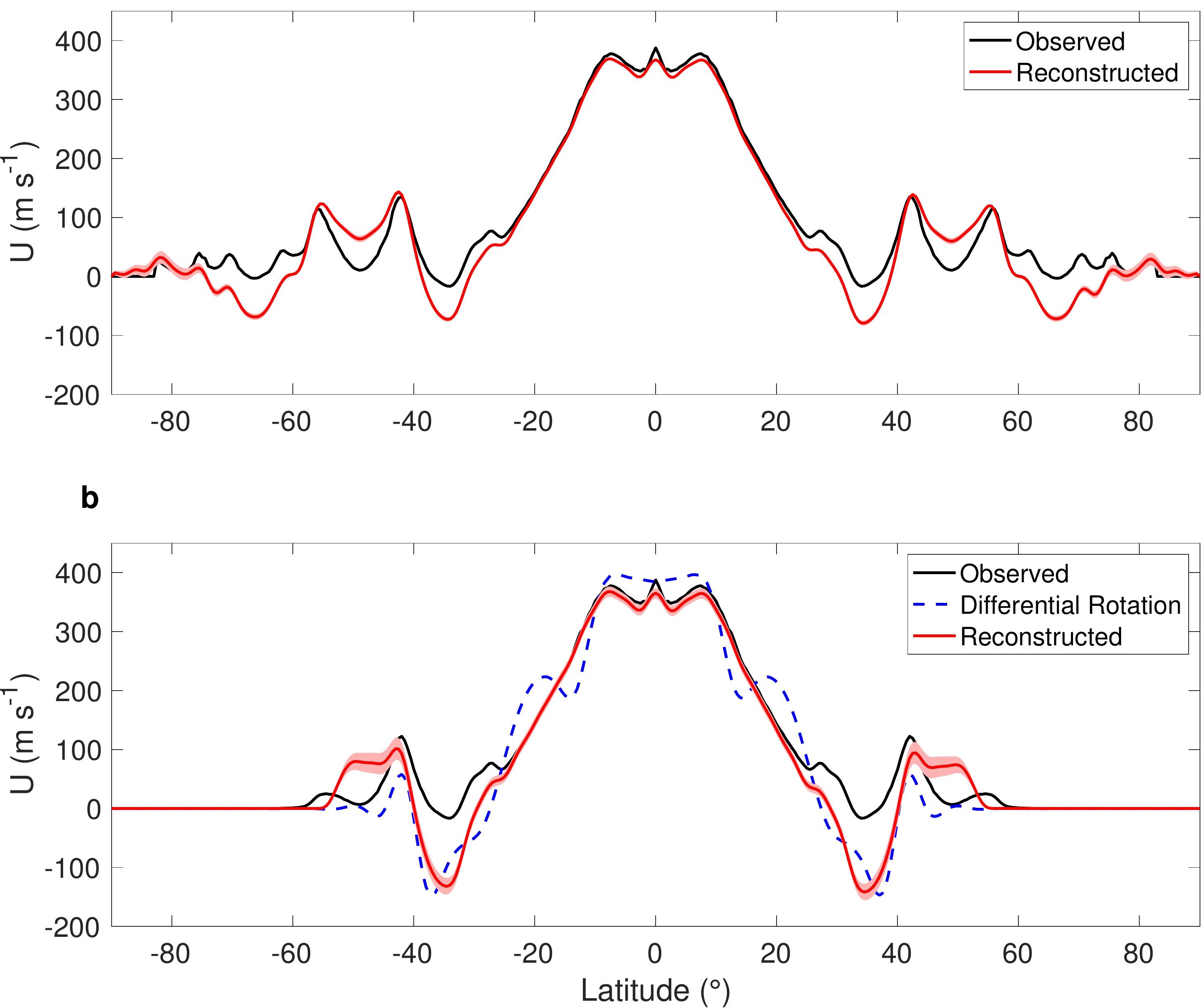




\section{Supplementary Materials for}

\section{Measurement and implications of Saturn's gravity field and ring mass}

Authors: L. Iess", B. Militzer, Y. Kaspi, P. Nicholson, D. Durante, P. Racioppa, A.

Anabtawi, E. Galanti, W. Hubbard, M. J. Mariani, P. Tortora, S. Wahl, M. Zannoni

*Correspondence to: luciano.iess@,uniroma1.it

\section{This PDF file includes:}

Materials and Methods

Figs. S1 to S6

Tables $\mathrm{S} 1$ to $\mathrm{S} 2$

References (70-73) 


\section{Materials and Methods}

Gravity pass selection and data pre-processing

The 22 Grand Finale orbits exhibit a variable range of altitudes above the Saturn surface due to the effect of distant Titan passes. The altitude above the reference 1-bar surface (equatorial radius $\sim 60280 \mathrm{~km}$ ) varies between roughly 1600 and $4000 \mathrm{~km}$. During the 4 highest orbits, the High Gain Antenna (HGA) was used to protect the spacecraft against possible ring particles from the very close $\mathrm{D}$ ring, thus preventing communication with Earth for much of the pass. Closest approach occurred just after the rings crossing (Table S1). These orbits were therefore not considered for gravity measurements. The last six orbits were also not considered, as the spacecraft flew through the upper layers of the planet's atmosphere. The large aerodynamic torque required thrusters to prevent tumbling, thus perturbing the orbital dynamics of the spacecraft.

Of the remaining 12 orbits, Revs 273,274, 275, 278, 280 and 284 were selected for gravity measurements. The selection criteria favored those with lower altitude and better coverage of the pericenter pass (in terms of higher tracking elevation from the ground stations), to maximize the projection of the Doppler signal, and minimize measurement noise caused by the Earth's troposphere.

The multi-arc fit uses 10649 observables in total, including $10116 \mathrm{X}$-band Doppler and 533 range observables. Among Doppler observables, 9950 are two-way and 166 are three-way. Many more three-way Doppler observables were acquired concurrently with two-way ones, but we used only a small fraction of the available three-way observables, in order to cover a gap on Rev 274 outbound caused by the $15^{\circ}$ elevation limit. These few data indirectly provide information on the gravity field because they allow a better estimation of the Cassini state vector for that arc. The selected count time for Doppler data was $30 \mathrm{~s}$, adequate to provide sufficient spatial sampling of the gravity field. 536 range observables are available in the same data span, but they provide only very limited information on the gravity field and therefore were not included in our solution

Ground stations acquired Doppler data with both closed loop and open loop receivers. The latter allow optimal pre-processing to minimize thermal noise in the reconstruction of the received signal frequency. We used open loop samples together with a reference trajectory (obtained by a preliminary fit to the closed loop observables) to pre-compensate for most of the signal dynamics. This data processing method provided a reduction of RMS residuals by up to a factor of 2 over closed loop samples at a count time of $1 \mathrm{~s}$.

Range measurements are affected by biases due to errors in the planetary ephemerides and instrumental effects. A small correction to Saturn's barycentric state vector is included in the estimate. This correction is expressed using the set III ephemeris parameters (70) and amounts to about $200 \mathrm{~m}$ relative to Earth, with respect to JPL's DE430 ephemerides (18). Residual range biases amount to less than $2.5 \mathrm{~m}$ and are compatible with the expected instrumental and media calibration error for the DSN ranging system. These biases are absorbed by ad-hoc local parameters (one for each participating station). 
Data analysis: additional information

In addition to 1-PN (Post Newtonian) relativistic effects accounted for in MONTE, we included also the 1.5-PN Lense-Thirring acceleration associated with Saturn's angular momentum, computed for a polar moment of inertia $C=0.26$ (as predicted by interior models) (1). The computation of the relativistic time delay included the contribution of Saturn's oblateness $\left(J_{2}\right)$.

Non-gravitational accelerations used in the orbital propagation included the solar radiation pressure and the thrust from the anisotropic thermal emission from the onboard power source (three radioisotope thermoelectric generators, radiating about $13 \mathrm{~kW}$ in total). This acceleration, constant in the spacecraft frame, was well determined by the navigation team during the mission's cruise and tour phases. Below $5000 \mathrm{~km}$ altitude the atmospheric drag was computed from a model of the neutral particle density in Saturn's upper atmosphere (mainly induced by molecular hydrogen) (71). The peak acceleration varied according to the altitude at closest approach, with a maximum of $3 \times 10^{-10} \mathrm{~m} \cdot \mathrm{s}^{-2}$ in Rev 274 (Table S1).

The data were adjusted to include the additional Doppler shift resulting from the effect of the spacecraft spin on the circularly polarized radio signal. This was largest during Rev 273 and Rev 284, when Cassini was spinning about the antenna axis to allow calibration of the magnetometer.

Solutions with a static tesseral field

A tesseral gravity field may be generated by atmospheric circulation, but it would likely be time variable on the time scale of the Grand finale orbits. Instead, a static field may appear if caused by deep convective flows in the planet, which have a longer time scale. A non-axisymmetric gravitational field arising from dynamo processes in the deep interior would also give rise to a tesseral field (72). Although a static tesseral field of high degree provides a good fit to the Doppler data, the degree and order of such a field depends on the assumed rotation rate.

The rotation rate of Saturn remains poorly known because different values have been inferred through different measurement methods. Magnetic field data from Voyager provided a period of $10 \mathrm{~h} 39 \mathrm{~m} 22 \mathrm{~s}$, also called System III (25). During the approach and first orbit of Saturn, the Cassini radio wave and plasma wave instrument investigation inferred a period of $10 \mathrm{~h} 45 \mathrm{~m} 45 \mathrm{~s}$ (26). Later measurements using the Cassini magnetometer estimated a slightly longer period of $10 \mathrm{~h} 47 \mathrm{~m} 6 \mathrm{~s}(27)$. A faster rotation of $10 \mathrm{~h} 32 \mathrm{~m} 45 \mathrm{~s}$ has been more recently derived from the measured gravity field (before the Grand Finale), observed shape, and possible internal density profiles (24).

Using each of these values to fit the Doppler data, we found that the required tesseral field varies from degree and order 8 to 12 (see legend in Fig. S4). The uncertainty in Saturn's rotation period precludes the use of the estimated tesseral coefficients to infer physical conditions inside Saturn. This does not apply to the zonal gravity coefficients and ring mass, whose estimates are statistically consistent across all of our gravity models (see Fig. S4 and Table S2). This gives us confidence in the ability of the reference solution, which uses a random acceleration model, to reproduce the zonal gravity. 
$\underline{\text { Solutions with normal modes }}$

An alternative Saturn gravity model assumes a time-variable field resulting from internal oscillation modes (28). Saturn's fundamental modes have been identified via density waves in the C ring (29), as observed in Cassini stellar occultation data. However, these observations led only to the determination of the azimuthal order, and no information was retrieved about the degree of the oscillations. The magnitude of the corresponding gravity coefficients has been estimated to be less than about $10^{-11}$ for those modes resonating with the rings (29).

In principle, normal modes can be included in the orbit determination process, with estimates of their amplitude and frequency. We found that several subsets of normal modes can fit the gravity data without the need of random accelerations acting on Cassini. But given the large number of possible modes, it is impossible to determine their characteristics uniquely with the available data, which makes the inclusion of any particular normal modes arbitrary.

To reduce the parameter space, we limited our analysis to normal modes with zonal symmetry. Normal modes of a tesseral nature are not included because their amplitudes, as estimated from ring seismology (29), are too small to be detected with Cassini. Concerning the type of modes to be included, we investigated pressure modes (f-modes and p-modes) as well as gravity modes (g-modes). The frequencies of the normal modes are adopted from (73). The Cassini Doppler data are insensitive to small errors in mode frequency due to the limited time span of the observations. We used three subsets of normal modes:

- f-modes: Only fundamental modes $\tilde{J}_{l, n=0}$ are estimated, from degree 2 to 10 ;

- f-modes and p-modes: Fundamental modes from degree 2 to 6 and the first few lowdegree, low-radial order p-modes (of degree 2 to 4 , first and second overtone);

- g-modes and f-modes: Gravity modes of degrees 2 and 3, with the inclusion of fundamental modes up to degree 6;

Each of these subsets can reduce the Doppler residuals to the noise level. However, the amplitudes of the normal modes are not well determined (statistically, they are compatible with zero amplitude), and thus no new information on the true values of the normal modes can be obtained with this analysis. Since our goal is to provide an unbiased estimate of Saturn's zonal harmonics and the ring mass, we instead show that the solutions obtained with different normal mode assumptions are compatible with our reference solution that employs random accelerations (see Fig. S4). 
(a)

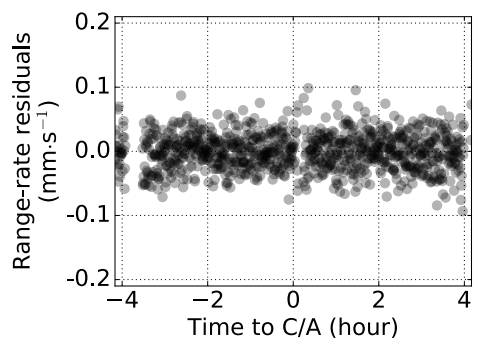

(d)

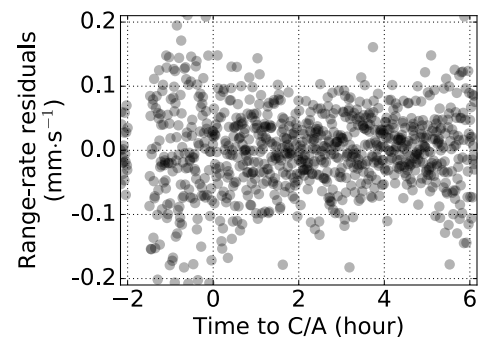

(b)

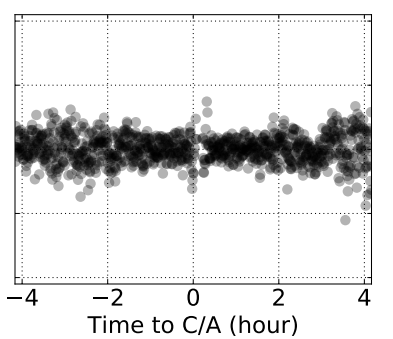

(e) (c)
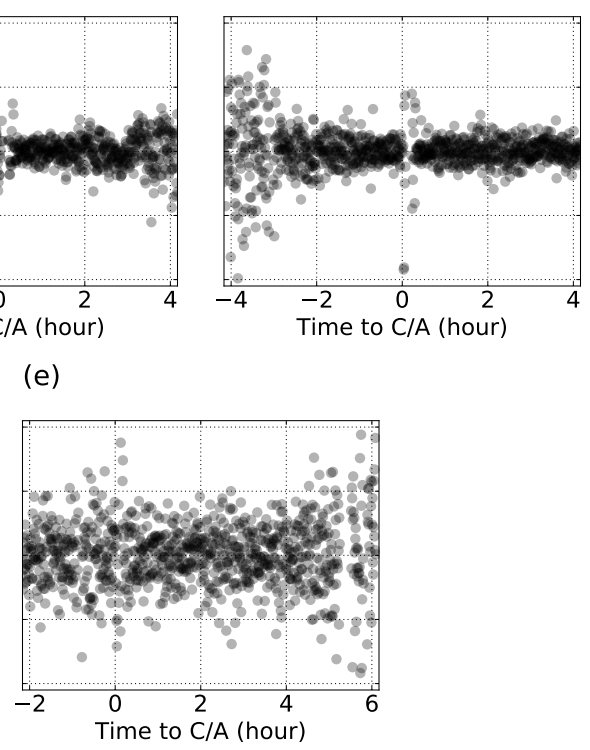

Fig. S1: Range rate residuals (integrated over $30 \mathrm{~s}$ ) for the pericentre passes.

(a) is $\operatorname{Rev} 273$, (b) is $\operatorname{Rev} 274$, (c) is $\operatorname{Rev} 278$, (d) is Rev 280, (e) is Rev 284. The noise RMS is reported in Table $\mathrm{S} 1$. 
(a)

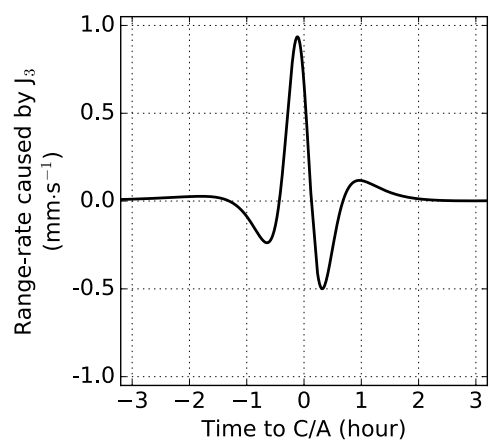

(b)

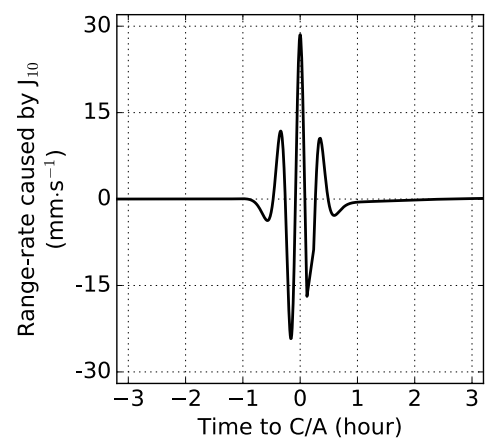

(c)

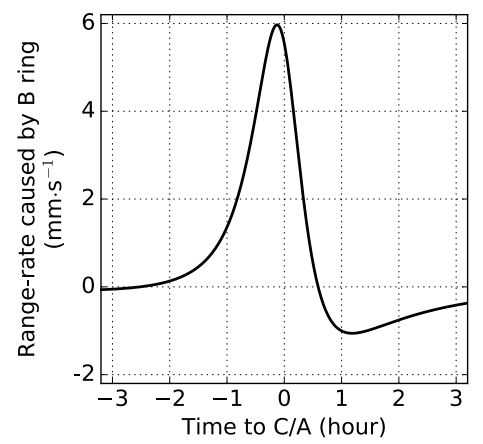

Fig. S2: Recovered range rate signals from (a) $\mathbf{J}_{3}$, (b) $\mathbf{J}_{10}$, and (c) $B$ ring mass for Rev 273. By comparison, the noise RMS for Rev273 is $0.029 \mathrm{~mm} / \mathrm{s}$ (see Table $\mathrm{S} 1$ ). 
(a)

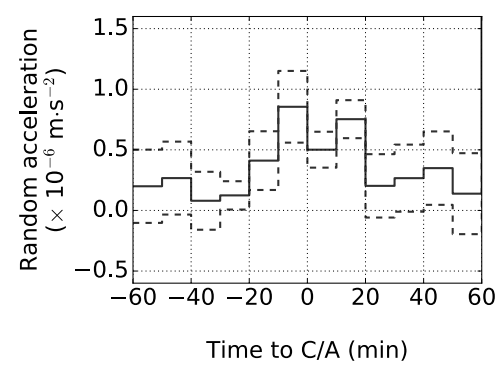

(d)

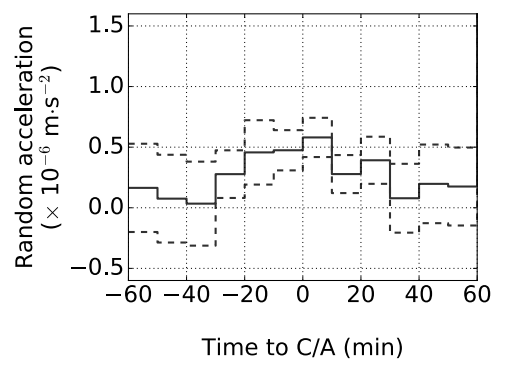

(b)

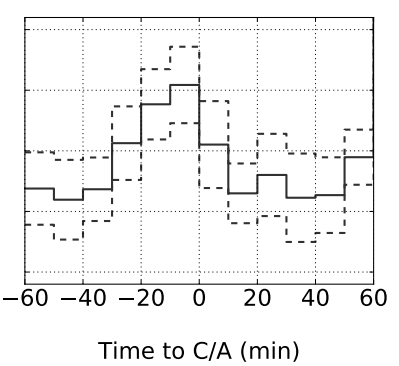

(c)

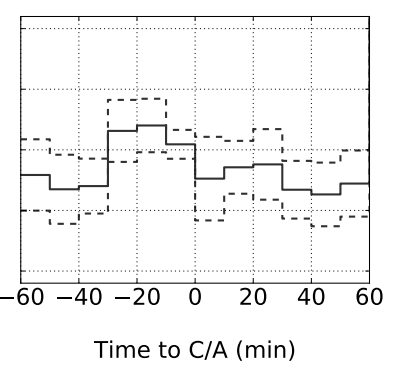

(e)

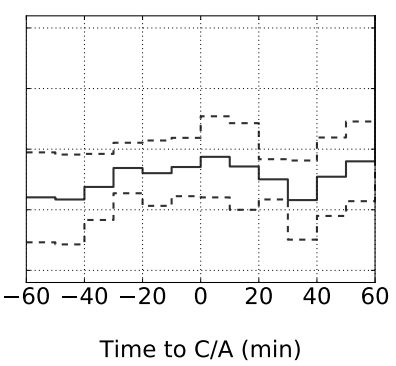

Fig. S3: Magnitude of the piecewise-constant acceleration needed to reduce residuals to the noise level. (a) is $\operatorname{Rev} 273$, (b) is Rev 274, (c) is Rev 278, (d) is Rev 280, (e) is Rev 284. The formal uncertainty (dashed lines) is reported along with the value (solid line). 
(a)

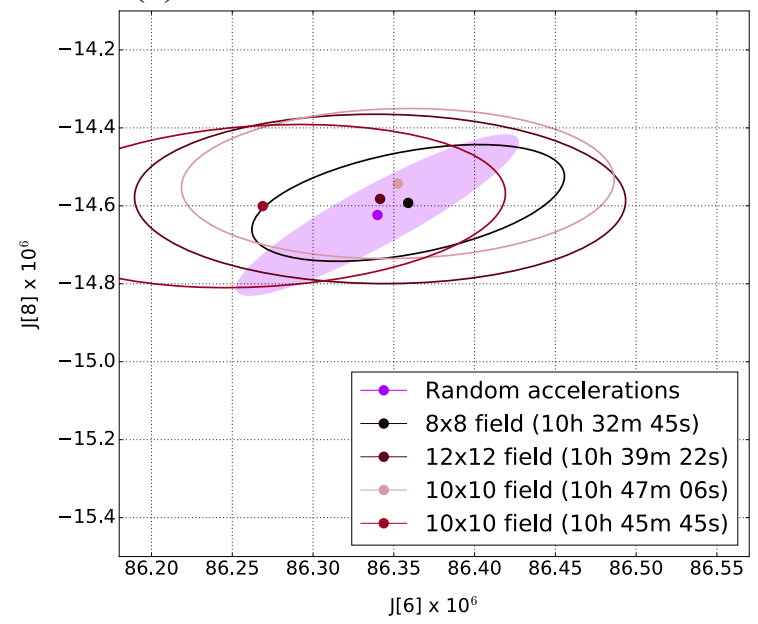

(c)

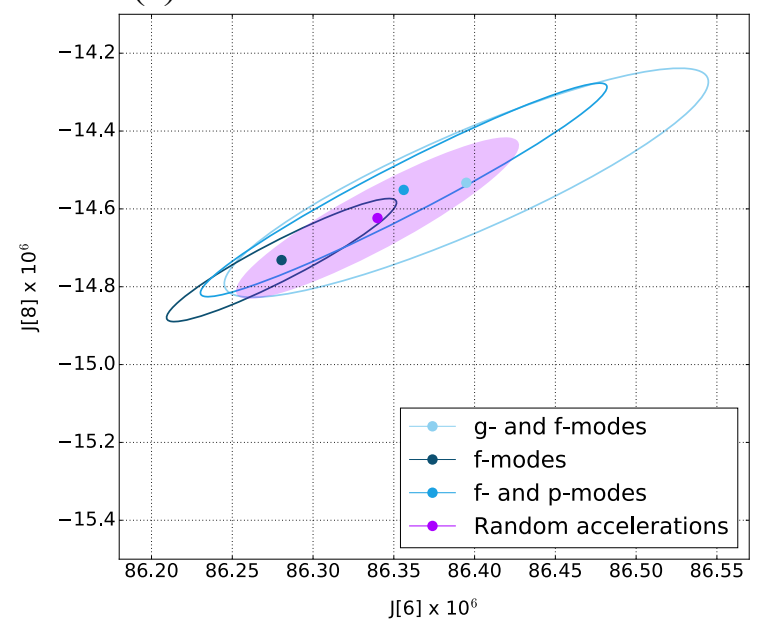

(b)

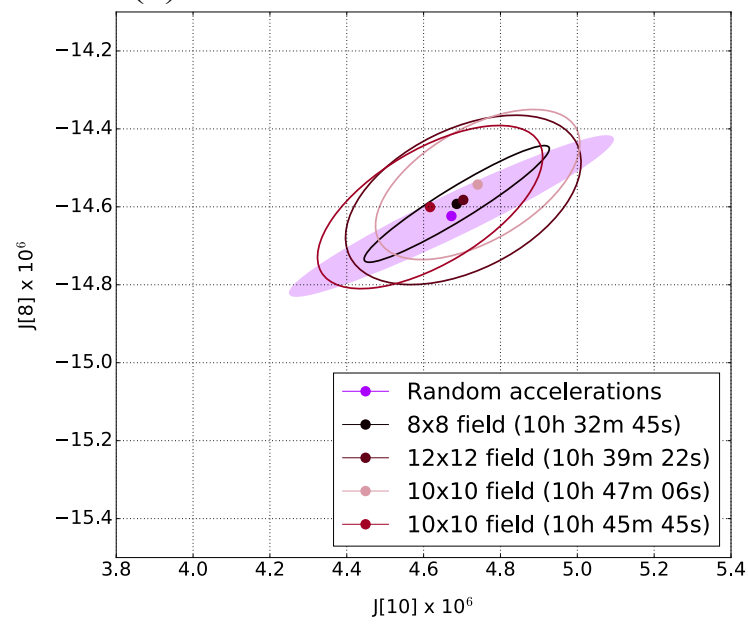

(d)

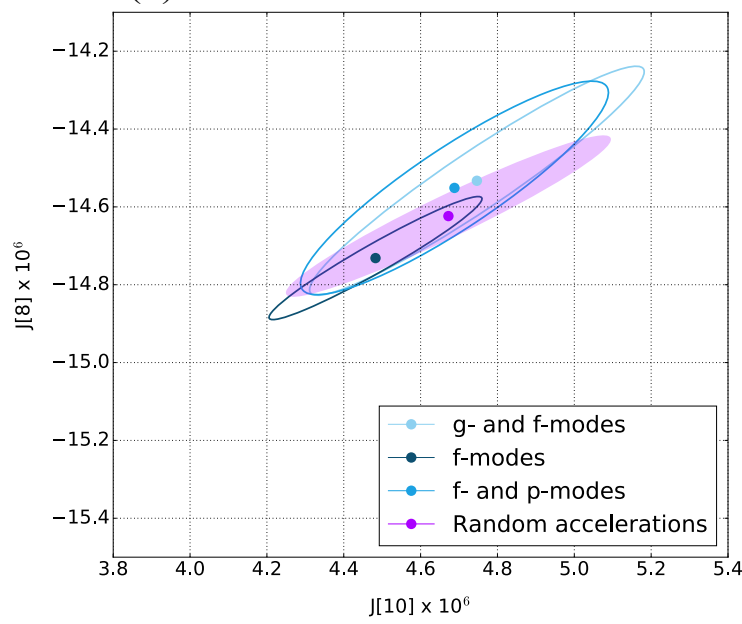

Fig. S4: $3 \sigma$ uncertainty ellipses in the $J_{6^{-}} J_{8}$ and $J_{10^{-}} J_{8}$ planes for different dynamical models. The random acceleration model is the reference solution (purple ellipse). The coefficients of the tesseral field (panels a and b) and normal mode (panels $c$ and d) models are constrained by an a-priori uncertainty $\left(1 \times 10^{-8}\right.$ and $3 \times 10^{-8}$ respectively) corresponding to the order of magnitude of the estimated random accelerations. For the tesseral model, the legend also reports the field degree and order corresponding to the minimum value required to obtain a good fit and the assumed Saturn rotation period. For the normal mode model, the legend also reports the type of modes assumed in each case. 


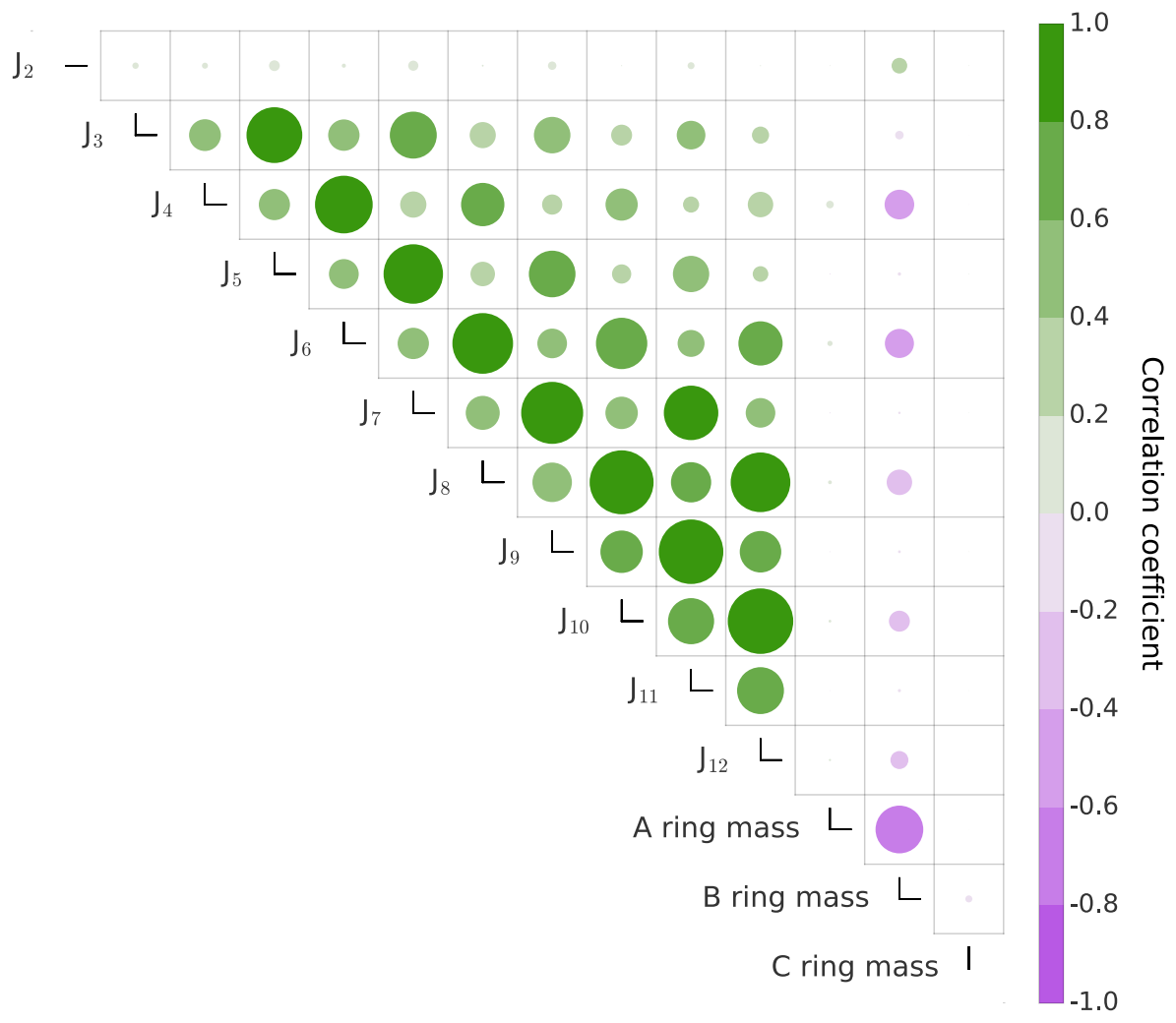

Fig. S5: Correlation matrix (upper triangular part). Correlation is reported for estimated zonal coefficients and ring masses in the reference solution obtained with random accelerations near $\mathrm{C} / \mathrm{A}$. The size and color of each circle is proportional to the correlation coefficient value (positive correlation in green tints, negative correlation in magenta tints). Adjacent even (or odd) zonal harmonics are strongly correlated except for $J_{2}$. The specific correlation pattern depends on the observation geometry and the a priori uncertainties. 


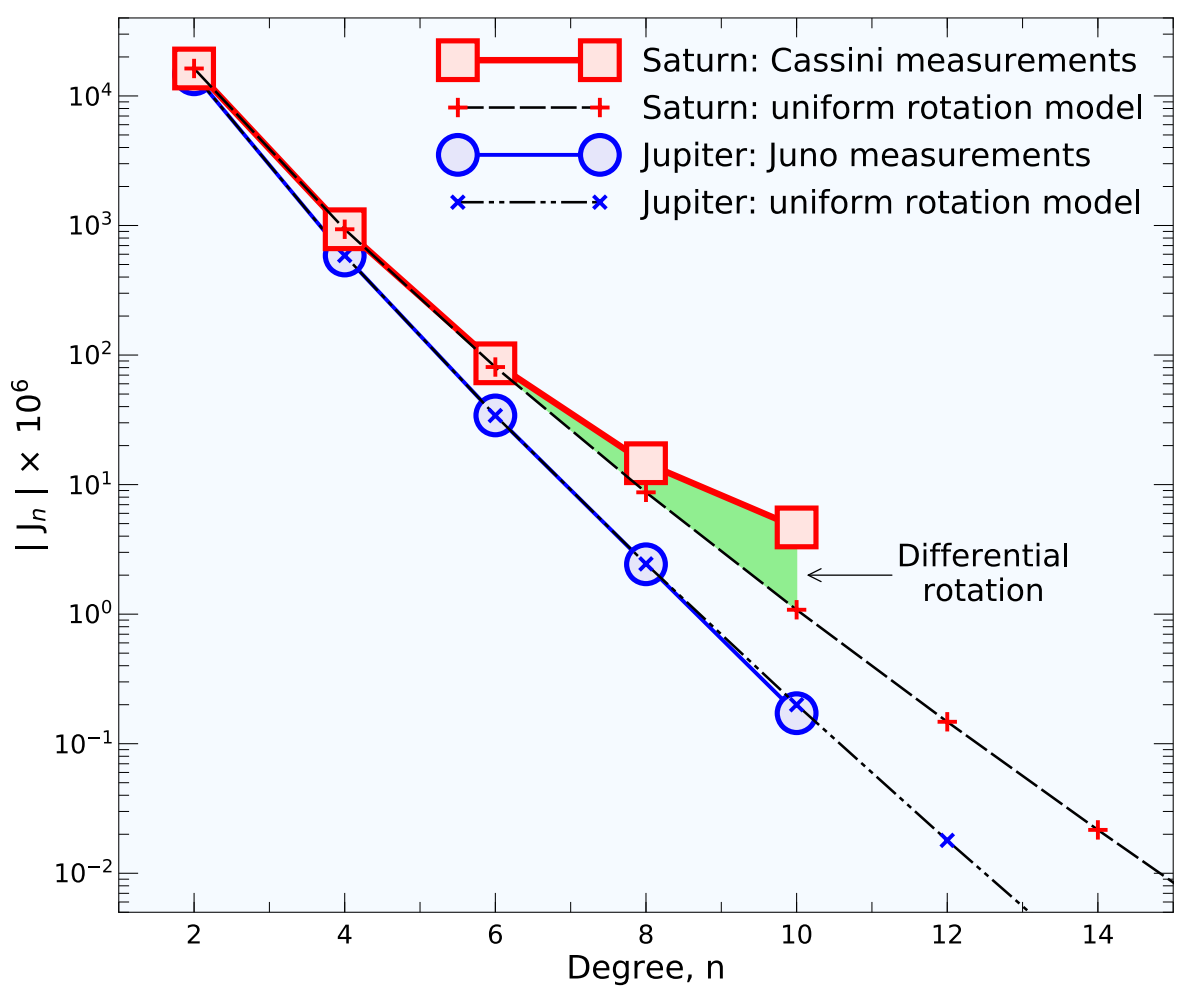

Fig. S6: Comparison of the even gravity harmonics of Saturn and Jupiter. The gravity coefficients have been measured respectively by the Cassini and Juno (5) spacecraft and compared with representative interior models that assume uniform rotation. While such models match Jupiter's gravitational field, for Saturn the diverging trends of the measurements and the uniform rotation models can be identified even on this logarithmic scale. The data require models with differential rotation (see Table 2). 
Table S1: Parameters of the osculating orbit at $\mathrm{C} / \mathrm{A}$ from the reconstructed trajectory of our best fitting solution. Orbit inclination and $\mathrm{C} / \mathrm{A}$ latitude are relative to the ring plane. The C/A altitude is relative to the Saturn 1-bar surface (equatorial radius of $60268 \mathrm{~km}$, flattening of 0.09796). We report also the Earth Boresight to Orbit normal (EBO) and SunEarth-Probe (SEP) angles. The reported noise level refers to the C/A pass only, for Doppler observables at $30 \mathrm{sec}$.

\begin{tabular}{|c|c|c|c|c|c|}
\hline Parameter & Rev 273 & Rev 274 & Rev 278 & Rev 280 & Rev 284 \\
\hline Eccentricity & 0.92 & 0.92 & 0.92 & 0.92 & 0.92 \\
\hline Inclination (deg) & 63 & 63 & 62 & 62 & 62 \\
\hline EBO (deg) & 96 & 96 & 98 & 99 & 101 \\
\hline C/A altitude $(\mathrm{km})$ & 2720 & 2668 & 3427 & 3409 & 2817 \\
\hline C/A latitude (deg) & -5 & -5 & -6 & -6 & -7 \\
\hline SEP $(\mathrm{deg})$ & 142 & 148 & 175 & 172 & 146 \\
\hline Noise RMS $(\mu \mathrm{m} / \mathrm{s})$ & 29 & 23 & 20 & 88 & 44 \\
\hline
\end{tabular}


Table S2: Estimates of the ring masses for different dynamical models. Values are reported for the stochastic, tesseral field, and normal modes solutions, in units of Mimas gravitational parameter $\left(G M_{M}=2.5026 \mathrm{~km}^{3} \cdot \mathrm{s}^{-2}\right)$. The uncertainties are $1 \sigma$. The solution with the tesseral field shows larger uncertainties due to its large number of free parameters. The reference solution (random accelerations) and the solution using normal modes are in good agreement. Although the masses of the three rings are highly correlated, their sum is well-determined and consistent at the $1 \sigma$ level.

\begin{tabular}{|c|c|c|c|c|}
\hline & A ring $\left(G M_{M}\right)$ & $\mathrm{B}$ ring $\left(G M_{M}\right)$ & $\mathrm{C}$ ring $\left(G M_{M}\right)$ & $\begin{array}{c}\mathrm{A}+\mathrm{B}+\mathrm{C} \text { rings } \\
\left(G M_{M}\right)\end{array}$ \\
\hline $\begin{array}{l}\text { A priori } \\
\text { uncertainty }\end{array}$ & 0.11 & 10.00 & 0.024 & 10.00 \\
\hline $\begin{array}{l}\text { Random } \\
\text { accelerations }\end{array}$ & $0.15 \pm 0.11$ & $0.23 \pm 0.20$ & $0.024 \pm 0.024$ & $0.41 \pm 0.13$ \\
\hline $\begin{array}{l}\text { Tesseral } 8 \times 8 \text { field } \\
(10 \mathrm{~h} 32 \mathrm{~m} 45 \mathrm{~s})\end{array}$ & $0.14 \pm 0.11$ & $0.16 \pm 0.31$ & $0.025 \pm 0.024$ & $0.32 \pm 0.27$ \\
\hline $\begin{array}{l}\text { Tesseral } 12 \times 12 \text { field } \\
(10 \mathrm{~h} 39 \mathrm{~m} 22 \mathrm{~s})\end{array}$ & $0.17 \pm 0.11$ & $0.10 \pm 0.46$ & $0.024 \pm 0.024$ & $0.29 \pm 0.43$ \\
\hline $\begin{array}{l}\text { Tesseral } 10 \times 10 \text { field } \\
(10 \mathrm{~h} 45 \mathrm{~m} 45 \mathrm{~s})\end{array}$ & $0.16 \pm 0.11$ & $0.43 \pm 0.37$ & $0.025 \pm 0.024$ & $0.62 \pm 0.34$ \\
\hline $\begin{array}{l}\text { Tesseral } 10 \times 10 \text { field } \\
(10 \mathrm{~h} 47 \mathrm{~m} 06 \mathrm{~s})\end{array}$ & $0.17 \pm 0.11$ & $0.16 \pm 0.38$ & $0.024 \pm 0.024$ & $0.35 \pm 0.35$ \\
\hline f-modes & $0.10 \pm 0.11$ & $0.37 \pm 0.15$ & $0.023 \pm 0.024$ & $0.49 \pm 0.07$ \\
\hline f- and $p$ - modes & $0.13 \pm 0.11$ & $0.29 \pm 0.18$ & $0.023 \pm 0.024$ & $0.45 \pm 0.12$ \\
\hline g- and $f$ - modes & $0.10 \pm 0.11$ & $0.36 \pm 0.18$ & $0.023 \pm 0.024$ & $0.48 \pm 0.13$ \\
\hline
\end{tabular}

